\title{
Sobre Um Problema de Perturbação Singular Com Vários Retardamentos
}

\author{
José Hilário DA CRUZ \\ Orientador: Prof. Dr. Plácido Zoega TÁboas
}

Tese apresentada ao Instituto de Ciências Matemáticas e de Computação, da Universidade de São Paulo, como parte dos requisitos para obtenção do título de Doutor em Ciências - Área: Matemática.

USP - Sāo Carlos

Maio de 1998 
Aos meus pais, minha esposa, meus filhos e ao Prof. Raimundo R. Ferreira (in memorian). 


\section{Resumo}

Consideremos a classe de equações diferenciais-diferenças singularmente perturbadas

$$
\varepsilon \dot{x}(t)=\sum_{r=0}^{l} \alpha_{r} x(t-r), \quad \varepsilon>0
$$

e seu limite formal quando $\varepsilon \rightarrow 0$ :

$$
0=\sum_{r=0}^{l} \alpha_{r} x(t-r) .
$$

Utilizando um método introduzido por Carvalho [5], exibimos soluções periódicas de $\left(1_{\varepsilon}\right)$ e $\left(1_{0}\right)$ e definimos hipersuperfícies de bifurcação dessas soluções no espaço dos parâmetros $\left(\alpha_{0}, \alpha_{1}, \ldots, \alpha_{l}\right)$.

Visando estabelecer relações entre as dinâmicas definidas por $\left(1_{\varepsilon}\right)$ e $\left(1_{0}\right)$, no caso $l=2, \alpha_{0}=-1$ provamos que a região de estabilidade de $\left(1_{\varepsilon}\right)$ no espaço $\left(\alpha_{1}, \alpha_{2}\right)$ aproxima a região de estabilidade de $\left(1_{0}\right)$, quando $\varepsilon \rightarrow 0$, num sentido definido precisamente no Teorema 4.1.1. 


$$
\begin{gathered}
\text { Abstract } \\
\varepsilon \dot{x}(t)=\sum_{r=0}^{l} \alpha_{r} x(t-r), \quad \varepsilon>0
\end{gathered}
$$

We consider the class of singularly perturbed differential-difference equations

and its formal limit as $\varepsilon \rightarrow 0$ :

$$
0=\sum_{r=0}^{l} \alpha_{r} x(t-r) .
$$

Using a method due to Carvalho [5], we exhibit periodic solutions of $\left(1_{\varepsilon}\right)$ and $\left(1_{0}\right)$ and define bifurcation hypersurfaces for these solutions in the parameter space $\left(\alpha_{0}, \alpha_{1}, \ldots, \alpha_{l}\right)$.

Aiming to establish relations between the dynamics of $\left(1_{\varepsilon}\right)$ and $\left(1_{0}\right)$ in case $l=2, \alpha_{0}=-1$, we prove that the stability region of $\left(1_{\varepsilon}\right)$ in the space $\left(\alpha_{1}, \alpha_{2}\right)$ approaches the stability region of $\left(1_{0}\right)$, as $\varepsilon \rightarrow 0$, in a precise sense given in Theorem 4.1.1. 


\section{Índice}

$\begin{array}{ll}\text { Introduçāo } & 1\end{array}$

1 Preliminares 5

1.1 Fatos Básicos ................. 5

1.1.1 Existência e Unicidade de Soluções . . . . . . . . . . 6

$1.2 U \mathrm{~m}$ Método para Obter Soluções Periódicas . . . . . . . . 7

1.2.1 Equações a Diferenças Lineares Autônomas . . . . . . . . 7

1.2.2 O Método de Carvalho . . . . . . . . . . . . 15

1.3 Estabilidade . . . . . . . . . . . . . . . . . 21

1.3.1 Equações Diferenciais a Diferenças . . . . . . . . . 23

1.3.2 Equações a Diferenças . . . . . . . . . . . . 26

2 Periodicidade em um Problema de Perturbaçāo Singular com Vários Retardamentos 31

2.1 O Problema . . . . . . . . . . . . . . . 31

2.2 Soluções Periódicas . . . . . . . . . . . . . . . 33

2.3 Hipersuperfícies de Bifurcação . . . . . . . . . . . . . 39

3 A Equaçāo Limite $\quad 48$

3.1 Soluções Periódicas . . . . . . . . . . . . . . 48

3.2 Um Caso em que Soluções são Periódicas . . . . . . . . . . . 53 
4 Um Estudo da Regiāo de Estabilidade 62

4.1 Estabilidade . . . . . . . . . . . . . . . . . . 63

$\begin{array}{ll}\text { Referências Bibliográficas } & \mathbf{7 0}\end{array}$ 


\section{Introdução}

Modelos usando equações diferenciais com retardamentos aparecem com muita frequência numa grande variedade de aplicações. Retardamentos no tempo são introduzidos em modelos matemáticos para descrever sistemas em que a relação causa e efeito não é simultânea, isto é, existe um lapso no tempo entre a ação e a reação. Beuter et al [2] estudaram certas doenças neurológicas usando modelos de equações diferenciais com dois retardamentos. Veja também [18] e, para inúmeros outros exemplos de aplicações, a referência [17].

Consideramos a equação

$$
\dot{y}(t)=F\left(y(t), y\left(t-r_{1}\right), y\left(t-r_{2}\right), \ldots, y\left(t-r_{l}\right)\right)
$$

onde $F$ é uma função de $\mathbb{R}^{l+1}$ em $\mathbb{R}$ e $l$ inteiro positvo.

Se $F(0, \ldots, 0)=0$ e $F$ é diferenciável, então (1) pode ser escrita na forma

$$
\dot{y}(t)=a_{0} y(t)+a_{1} y\left(t-r_{1}\right)+\cdots+a_{l} y\left(t-r_{l}\right)+h\left(y(t), y\left(t-r_{1}\right), \ldots, y\left(t-r_{l}\right)\right),
$$

onde $a_{j}:=D_{j} F(0, \ldots, 0)$ é a derivada de $F$ na origem com relação à $j$-ésima variável, $j=0,1, \ldots, l \mathrm{e}$

$$
h\left(y_{0}, y_{1}, \ldots, y_{l}\right)=F\left(y_{0} \ldots, y_{l}\right)-\sum_{j=0}^{l} a_{j} y_{j}
$$

Como a equação não linear (1) herda importantes propriedades da equação 
linear

$$
\dot{y}(t)=a_{0} y(t)+\sum_{j=1}^{l} a_{j} y\left(t-r_{j}\right), \quad l \geq 1, \quad r_{j}>0
$$

o estudo de (2) tem grande interesse, ver Hale [17].

No Capítulo 1 apresentamos alguns fatos básicos para a apresentação desse trabalho. $\mathrm{Na}$ seção 1.2.2, descrevemos sucintamente um método introduzido por Carvalho em [5], que tem sido bastante utilizado, ver [7], [8] e referências ali contidas, onde é considerada uma equação de evolução do tipo

$$
\dot{x}(t)=f\left(\alpha, t, x_{t}\right), \quad t \geq \sigma
$$

onde $f: \mathbb{R}^{k+1} \times C \rightarrow \mathbb{R}$ é uma aplicação contínua, $\alpha \in \mathbb{R}^{k}$ é um parâmetro, $C$ é o espaço das funções contínuas $\varphi:[-1,0] \rightarrow \mathbb{R}$, munido da norma do supremo $\|\cdot\|$, $\sigma$ é um número real dado e se, $x:[\sigma-1, b] \rightarrow \mathbb{R}, b>\sigma$, é uma função contínua, $x_{t} \in C, \sigma \leq t \leq b$, é a aplicação definida por

$$
x_{t}(\theta)=x(t+\theta), \quad-1 \leq \theta \leq 0
$$

Dada $\varphi \in C$, o problema de valor inicial para a equação (3) é o sistema formado por (3) e a condição

$$
x_{\sigma}(\theta)=\varphi(\theta), \quad \theta \in[-1,0]
$$

Quando necessário, denotamos por $x(\cdot, \sigma, \varphi)$ uma solução do problema de valor inicial (3)-(4). Uma tal solução é periódica de período $p$ se $x(t+p, \sigma, \varphi)=x(t, \sigma, \varphi)$ para todo $t \in \mathbb{R}$. Assim, soluções periódicas são definidas para todo $t$. Para abreviar, a frase "periódica de período $p$ " será substituida por " $p$-periódica".

O método também se aplica naturalmente a equações do tipo

$$
F\left(t, x\left(t-r_{0}\right), \ldots, x\left(t-r_{l}\right)\right)=0
$$


onde $F$ é uma função contínua de $l+2$ variáveis, conhecidas como Equações a Diferenças. Atualmente, tem crescido de forma significativa o interesse nas equações a diferenças, tanto na versão contínua $(t \in \mathbb{R})$ quanto na discreta $(t \in \mathbb{Z})$, ver [22], [26], [30].

Mesmo as equações a diferenças mais simples, como as lineares.

$$
x(t)=\sum_{i=0}^{l} a_{i} x\left(t-r_{i}\right),
$$

apresentam fenômenos de alta sensibilidade com relação às condições iniciais e parâmetros.

Por exemplo, nas aplicações os números $r_{i}$ 's são os retardamentos do sistema, tais como tempo de comunicação entre dois circuitos elétricos, tempo de resposta em sistemas de controle com "feedback". O problema é que nas aplicações as condições iniciais, os retardamentos e os parâmetros nem sempre podem ser medidos com precisão. Assim, é importante conhecer os efeitos de pequenas perturbações dessas medidas nas propriedades qualitativas do sistema.

Num sistema "bem comportado" esperamos que estas propriedades se preservem sob pequenas perturbações das condições iniciais, retardamentos ou parâmetros. Melvin [26] ilustra o fato que, em geral, equações do tipo (6) não são "bem comportadas" com relação aos retardamentos.

No Capítulo 2, aplicamos o método para estudar a localização das $(l+1)$-uplas $\left(\alpha_{0}, \ldots, \alpha_{l}\right)$, para as quais exibimos alguns tipos de soluções periódicas da equação

$$
\varepsilon \dot{x}(t)=\sum_{r=0}^{l} \alpha_{r} x(t-r), \quad \varepsilon>0 .
$$

Definimos também as hipersuperfícies de bifurcação, explicitando suas parametrizações.

No Capítulo 3, obtivemos soluções periódicas para o caso singular $(\varepsilon=0)$ e mais algumas informações tais como condições para que todas as soluções sejam periódicas com um mesmo período. 
Finalmente, no Capítulo 4, fizemos um estudo e obtivemos algumas informações sobre a região de estabilidade no espaço dos parâmetros $\left(\alpha_{1}, \alpha_{l}\right)$, para $\circ$ caso $\alpha_{0}=-1, \alpha_{2}=\cdots=\alpha_{l-1}=0$, isto é, para a equação

$$
\varepsilon \dot{x}(t)+x(t)=\alpha_{1} x(t-1)+\alpha_{l} x(t-l)
$$

onde $l \geq 2$ é um inteiro. Para o caso $l=2$, mostramos que esta região aproxima da região de estabilidade a equação

$$
x(t)=\alpha_{1} x(t-1)+\alpha_{2} x(t-2)
$$

quando $\varepsilon \rightarrow 0$, num sentido definido precisamente no Teorema 4.1.1. 


\section{Capítulo 1}

\section{Preliminares}

Uma teoria fundamental para as Equações Diferenciais Funcionais Retardadas pode ser encontrada em [17], com versões mais gerais do Teorema 1.1 .1 e do Teorema 1.1.2 a seguir, incluindo prolongamento de soluções e continuidade com relação às condições iniciais e parâmetros. Admitimos tacitamente o contexto de [17] no que concerne às notações e fatos desta teoria. Visando tornar a leitura tanto quanto possível independente, damos abaixo alguns fatos fundamentais dessa teoria, para a apresentação desse trabalho.

\subsection{Fatos Básicos}

Representaremos por $C\left([a, b], \mathbb{R}^{n}\right)$ o espaço de Banach, das funções contínuas aplicando o intervalo real $[a, b]$ em $\mathbb{R}^{n}$, com a topologia da convergência uniforme, isto é, para $\varphi \in C\left([a, b], \mathbb{R}^{n}\right)$, a norma é definida como

$$
\|\varphi\|=\sup _{a \leq \theta \leq b}|\varphi(\theta)|
$$

onde $|\cdot|$ é uma norma do $\mathbb{R}^{n}$.

Quando $[a, b]=[-r, 0]$, onde $r$ é uma constante positiva, definimos $C:=$ $C\left([-r, 0], \mathbb{R}^{n}\right)$. Para $\sigma, A \in \mathbb{R}, A \geq 0, x \in C\left([\sigma-r, \sigma+A], \mathbb{R}^{n}\right)$ e $t \in[\sigma, \sigma+A]$ 
indicamos $x_{t} \in C$, como é usual, dada por

$$
x_{t}(\theta)=x(t+\theta), \quad \theta \in[-r, 0]
$$

Dado um subconjunto aberto, $\Omega$, de $\mathbb{R} \times C$ e uma função $f: \Omega \rightarrow \mathbb{R}^{n}$, chamamos a equação

$$
\dot{x}(t)=f\left(t, x_{t}\right)
$$

de uma Equação Diferencial Funcional Retardada em $\Omega$. Uma função $x$ é chamada solução de (1.1) em $[\sigma-r, \sigma+A)$ se $x \in C\left([\sigma-r, \sigma+A), \mathbb{R}^{n}\right),\left(t, x_{t}\right) \in \Omega$ e $x_{t}$ satisfaz (1.1) para $t \in[\sigma, \sigma+A)$.

Para $(\sigma, \varphi) \in \Omega$, dizemos que $x(t ; \sigma, \varphi)$ é uma solução de $(1.1)$ com valor inicial $(\sigma, \varphi)$, se existir um $A>0$ tal que $x(t ; \sigma, \varphi)$ satisfaz $(1.1)$ em $[\sigma-r, \sigma+A)$ e $x_{\sigma}(\varphi)=\varphi$.

Observe que encontrar uma solução de (1.1) passando por $(\sigma, \varphi) \in \Omega$ é equivalente a resolver a equação integral

$$
\begin{aligned}
x(t) & =\varphi(0)+\int_{\sigma}^{t} f\left(s, x_{s}\right) d s, \quad t \geq \sigma, \\
x_{\sigma} & =\varphi .
\end{aligned}
$$

\subsubsection{Existência e Unicidade de Soluções}

Para provar a existência de solução passando por $(\sigma, \varphi)$, pode-se proceder de maneira análoga ao da teoria fundamental das Equações Diferenciais Ordinárias considerando um operador $T$ em $C_{\alpha}^{\sigma}:=C\left([\sigma-r, \alpha], \mathbb{R}^{n}\right), \alpha>\sigma$, definido por

$$
T y(t)=\varphi(0)+\int_{\sigma}^{t} f\left(s, y_{s}\right) d s, \quad \sigma \leq t \leq \alpha ; \quad\left(T y_{\sigma}\right)=\varphi .
$$

Para um conveniente subconjunto limitado, fechado e convexo, $\mathcal{A}$ de $C_{\alpha}^{\sigma}$ mostra-se que $T \mathcal{A} \subset \mathcal{A}$. Assim, segue de uma aplicação do Teorema de Schauder a versão abaixo do Teorema de Peano. 
Teorema 1.1.1. Em (1.1), suponha que $\Omega$ seja um subconjunto aberto de $\mathbb{R} \times C$ e $f$ contínua em $\Omega$. Se $(\sigma, \varphi) \in \Omega$, então existe uma solução de (1.1) passando por $(\sigma, \varphi)$, definida num intervalo $[\sigma-r, \sigma+A)$, para algum $A>0$.

Para garantir a unicidade, precisamos exigir mais da função $f$.

Dizemos que $f(t, \varphi)$ é Lipschitz em $\varphi$ em cada conjunto compacto $K$ de $\mathbb{R} \times C$ se existir uma constante $k>0$ tal que, para qualquer $\left(t, \varphi_{1}\right),\left(t, \varphi_{2}\right) \in K$,

$$
\left|f\left(t, \varphi_{1}\right)-f\left(t, \varphi_{2}\right)\right| \leq k\left|\varphi_{1}-\varphi_{2}\right| .
$$

Teorema 1.1.2. Sejam $\Omega$ um subconjunto aberto de $\mathbb{R} \times C$ ef $f \in C\left(\Omega, \mathbb{R}^{n}\right)$, e $f(t, \varphi)$ for Lipschitz em $\varphi$ em cada conjunto compacto de $\Omega$. Se $(\sigma, \varphi) \in \Omega$, então existe uma única solução de (1.1) passando por $(\sigma, \varphi)$, definida num intervalo $[\sigma-r, \sigma+A)$, para algum $A>0$.

\subsection{Um Método para Obter Soluçōes Periódicas}

Nesta seção descreveremos, sucintamente, um método para a investigação de soluções periódicas originalmente apresentado em [5] para equações diferenciais com retardamento, embora ele se aplique naturalmente também no contexto das equações a diferenças.

As provas são apresentadas por razões de independência. A subseção que se segue é fundamental para a prova do principal resultado deste método.

\subsubsection{Equações a Diferenças Lineares Autônomas}

Uma equação a diferença linear autônoma é uma equação da forma:

$$
X(t)=A X(t-1)
$$

onde $X$ é uma função real a valores em $\mathbb{R}^{k}, k$ inteiro positivo, $A$ uma matriz $k \times k$, real ou complexa. 
Note que se $a_{1}(t), a_{2}(t)$ são funções escalares 1-periódicas quaisquer e $X_{1}(t)$ e $X_{2}(t)$ são soluções de (1.4), então

$$
X(t)=a_{1}(t) X_{1}(t)+a_{2}(t) X_{2}(t)
$$

também é solução de (1.4).

Observe que nas equações a diferenças lineares as funções escalares 1-periódicas ocupam o papel desempenhado pelas constantes em equações diferenciais lineares. Baseado neste fato, introduziremos a seguinte definição.

Definiçāo 1.2.1. As funções $X_{1}(t), X_{2}(t), \ldots, X_{n}(t), n \geq 1$ são linearmente dependentes sobre as funções 1-periódicas num intervalo $I$, ou simplesmente l.d., se existirem um conjunto $J \subset I$ de medida não nula e funções escalares 1-periódicas, $a_{1}(t), \ldots, a_{n}(t)$, tais que

$$
\sum_{j=1}^{n}\left|a_{j}(t)\right|^{2}>0 \quad \text { em } J \quad \text { e } \sum_{j=1}^{n} a_{j}(t) X_{j}(t)=0 \quad \text { q.s. em } I,
$$

onde a abreviação q.s. (quase sempre) significa que a igualdade vale para $t$ pertencente ao intervalo I exceto em um subconjunto de medida nula.

Se quaisquer funçôes escalares $a_{j}$ 's, 1-periódicas, que satisfazem a segunda condição de (1.5) forem nulas q.s. em $I$, dizemos que $X_{1}(t), \ldots, X_{n}(t)$ são linearmente independentes em I sobre as funçôes 1-periódicas, ou simplemente l.i.

Lema 1.2.1. Quaisquer soluções $X_{1}(t), X_{2}(t), \ldots, X_{k+1}(t)$ de (1.4) definidas para $t \geq t_{0}$ são l.d. em $\left[t_{0}, \infty\right)$.

Prova. Observe que para cada $t$, os vetores $X_{1}(t), X_{2}(t), \ldots, X_{k+1}(t) \in \mathbb{R}^{k}$ são l.d., então existem escalares $a_{1}(t), a_{2}(t), \ldots, a_{k+1}(t)$ tais que

$$
\sum_{j=1}^{k+1}\left|a_{j}(t)\right|^{2}>0 \quad \text { e } \quad \sum_{j=1}^{k+1} a_{j}(t) X_{j}(t)=0, \quad t \in\left[t_{0}, t_{0}+1\right) .
$$


Estendemos $a_{j}(t)$ sobre $\mathbb{R}, j=1, \ldots, k+1$, impondo 1-periodicidade. Assim, tomando $J=\left[t_{0}, t_{0}+1\right) \subset\left[t_{0}, \infty\right)$, resta apenas provar que $\sum_{j=1}^{k+1} a_{j}(t) X_{j}(t)=0$, q.s. em $\left[t_{0}, \infty\right)$.

Temos que

$$
\begin{aligned}
\sum_{j=1}^{k+1} a_{j}(t+1) X_{j}(t+1) & =\sum_{j=1}^{k+1} a_{j}(t) X_{j}(t+1) \\
& =\sum_{j=1}^{k+1} a_{j}(t) A X_{j}(t) \\
& =A \sum_{j=1}^{k+1} a_{j}(t) X_{j}(t)=0,
\end{aligned}
$$

para todo $t \in\left[t_{0}, t_{0}+1\right)$. Isto mostra que $X_{1}(t), \ldots, X_{k+1}(t)$ são l.d. em $\left[t_{0}, \infty\right)$.

Lema 1.2.2. Sejam $X_{1}(t), X_{2}(t), \ldots, X_{k}(t)$ soluções l.i. de (1.4), definidas para $t \geq t_{0}$. Então, qualquer outra solução pode ser escrita, de modo único, na forma

$$
X(t)=\sum_{j=1}^{k} a_{j}(t) X_{j}(t), \quad t \geq t_{0},
$$

onde $a_{j}$ 's são funções 1-periódicas.

Prova. Seja $X(t)$ uma solução de (1.4). Daí, existem escalares únicos, q.s., $a_{1}(t), a_{2}(t), \ldots, a_{k}(t)$ tais que

$$
X(t)=\sum_{j=1}^{k} a_{j}(t) X_{j}(t), \quad \text { q.s. em }\left[t_{0}, t_{0}+1\right) .
$$

Definamos $a_{j}(t+1)=a_{j}(t)$, para $t \in\left[t_{0}, t_{0}+1\right)$. Dessa forma, $a_{j}(t)$ está definida para $t \in\left[t_{0}, t_{0}+2\right)$ e, pela linearidade,

$$
Y(t)=\sum_{j=1}^{k} a_{j}(t) X_{j}(t), \quad \text { para } \quad t \in\left[t_{0}, t_{0}+2\right),
$$

também é solução de (1.4). 
Como $Y(t)$ e $X(t)$ coincidem em $\left[t_{0}, t_{0}+1\right)$, pela unicidade de solução de uma equação a diferença, tomando como função inicial $X(t)$ em $\left[t_{0}, t_{0}+1\right)$, temos que $Y(t)=X(t)$ em $\left[t_{0}, t_{0}+2\right)$. Assim, $X(t)=\sum_{j=1}^{k} a_{j}(t) X_{j}(t)$ para $t \in\left[t_{0}, t_{0}+2\right)$. Repetindo o processo, o lema fica provado.

Lema 1.2.3. Se $e^{b i}$ é um autovalor de $A, b \in \mathbb{R}$, com autovetor $v$, então

$$
\begin{aligned}
& X_{1}(t)=\Re(v) \cos [b(t+1)]-\Im(v) \operatorname{sen}[b(t+1)] \quad e \\
& X_{2}(t)=\Re(v) \operatorname{sen}[b(t+1)]+\Im(v) \cos [b(t+1)],
\end{aligned}
$$

são soluções de (1.4).

Além disso, se $b=k \pi, k \in \mathbb{Z}$, então $X_{1}(t)$ e $X_{2}(t)$ são l.d. (l.i.) se $\Re(v)$ e $\Im(v)$ forem l.d. (l.i.). Se $b \neq k \pi, k \in \mathbb{Z}$, então $X_{1}(t)$ e $X_{2}(t)$ são l.i..

Prova. Como $A v=e^{b i} v$, temos

$$
\begin{aligned}
& A \Re(v)=\Re(v) \cos b-\Im(v) \operatorname{sen} b, \\
& A \Im(v)=\Im(v) \cos b+\Re(v) \operatorname{sen} b .
\end{aligned}
$$

Assim,

$$
\begin{aligned}
A X_{1}(t-1) & =A[\Re(v) \cos (b t)-\Im(v) \operatorname{sen}(b t)] \\
& =A \Re(v) \cos (b t)-A \Im(v) \operatorname{sen}(b t) \\
& =[\Re(v) \cos b-\Im(v) \operatorname{sen} b] \cos (b t)-[\Im(v) \cos b+\Re(v) \operatorname{sen} b] \operatorname{sen}(b t) \\
& =\Re(v) \cos [b(t+1)]-\Im(v) \operatorname{sen}[b(t+1)] \\
& =X_{1}(t) .
\end{aligned}
$$

Portanto, $X_{1}(t)$ é solução de (1.4). De forma análoga podemos provar que $X_{2}(t)$ também é.

Agora, se $b=k \pi$, então podemos escrever

$$
\operatorname{sen}(b t)=a(t) \cos (b t)
$$


onde $a(t)=\tan (b t)$, que é 1-periódica.

Assim,

$$
\begin{aligned}
X_{1}(t) & =\Re(v) \cos [b(t+1)]-\Im(v) \operatorname{sen}[b(t+1)] \\
& =\Re(v) \cos (b t) \cos b-\Im(v) \operatorname{sen}(b t) \cos b \\
& =\Re(v) \cos (b t) \cos b-a(t) \Im(v) \cos (b t) \cos b \\
& =[\Re(v)-a(t) \Im(v)] \cos (b t) \cos b,
\end{aligned}
$$

e

$$
\begin{aligned}
X_{2}(t) & =\Re(v) \operatorname{sen}[b(t+1)]+\Im(v) \cos [b(t+1)] \\
& =\Re(v) \operatorname{sen}(b t) \cos b+\Im(v) \cos (b t) \cos b \\
& =a(t) \Re(v) \cos (b t) \cos b+\Im(v) \cos (b t) \cos b \\
& =[a(t) \Re(v)+\Im(v)] \cos (b t) \cos b .
\end{aligned}
$$

Temos dois casos a considerar:

i) Se $\Re(v)$ e $\Im(v)$ são l.d., então temos dois subcasos:

$\left.i_{1}\right) \Re(v)=c \Im(v), c \in \mathbb{R}$ e $\Im(v) \neq 0$, ou

$\left.i_{2}\right) \Im(v)=d \Re(v), d \in \mathbb{R}$ e $\Re(v) \neq 0$.

Para o subcaso $\left(i_{1}\right)$, temos

$$
\begin{aligned}
& X_{1}(t)=[c-a(t)] \Im(v) \cos (k \pi t) \cos (k \pi), \\
& X_{2}(t)=[c a(t)+1] \Im(v) \cos (k \pi t) \cos (k \pi) .
\end{aligned}
$$

Daí,

$$
X_{2}(t)=\frac{c a(t)+1}{c-a(t)} X_{1}(t), \quad a(t)=\tan (b t) .
$$

Portanto, $X_{1}(t)$ e $X_{2}(t)$ são l.d..

O subcaso $\left(i_{2}\right)$ é análogo. 
ii) Se $\Re(v)$ e $\Im(v)$ são l.i.. Observe que

$$
\begin{aligned}
a_{1}(t) X_{1}(t)+a_{2}(t) X_{2}(t)= & a_{1}(t)[(\Re(v)-a(t) \Im(v)) \cos (b t) \cos b]+ \\
& +a_{2}(t)[(\Re(v) a(t)+\Im(v)) \cos (b t) \cos b] \\
= & \cos (b t) \cos b\left[\left(a_{1}(t)+a_{2}(t) a(t)\right) \Re(v)+\right. \\
& \left.+\left(a_{2}(t)-a_{1}(t) a(t)\right) \Im(v)\right] \\
= & {\left[a_{1}(t)+a_{2}(t) a(t)\right] \Re(v)+\left[a_{2}(t)-a_{1}(t) a(t)\right] \Im(v) . }
\end{aligned}
$$

Como $\Re(v)$ e $\Im(v)$ são l.i. temos que

$$
a_{1}(t) X_{1}(t)+a_{2}(t) X_{2}(t)=0
$$

implica

$$
a_{1}(t)+a_{2}(t) a(t)=0 \quad \text { e } \quad a_{2}(t)-a_{1}(t) a(t)=0
$$

isto é,

$$
a_{1}(t), a_{2}(t) \equiv 0
$$

Isto completa a prova no caso $b=k \pi, k \in \mathbb{Z}$.

Agora, se $b \neq k \pi$ para todo $k \in \mathbb{Z}$, então $\cos (b t)$ e $\operatorname{sen}(b t)$ são l.i.. De fato, se

$$
a_{1}(t) \cos (b t)+a_{2}(t) \operatorname{sen}(b t)=0
$$

então

$$
a_{1}(t+1) \cos [b(t+1)]+a_{2}(t+1) \operatorname{sen}[b(t+1)]=0,
$$

isto é,

$$
\left[a_{1}(t) \cos b-a_{2}(t) \operatorname{sen} b\right] \operatorname{sen}(b t)+\left[a_{1}(t) \operatorname{sen} b+a_{2}(t) \cos b\right] \cos (b t)=0 .
$$


Logo, $Y(t)=\operatorname{col}(\operatorname{sen}(b t), \cos (b t))$ é uma solução não trivial do sistema

$$
A Y(t)=0
$$

onde

$$
A=\left[\begin{array}{cc}
a_{1}(t) & a_{2}(t) \\
a_{1}(t) \cos b-a_{2}(t) \operatorname{sen} b & a_{1}(t) \operatorname{sen} b+a_{2}(t) \cos b
\end{array}\right] .
$$

Portanto, o determinante da matriz do sistema é igual a zero, isto é,

$$
\left[a_{1}^{2}(t)+a_{2}^{2}(t)\right] \operatorname{sen} b=0,
$$

o que implica, $a_{1}(t), a_{2}(t) \equiv 0$.

Observe que

$$
\begin{aligned}
a_{1}(t) X_{1}(t)+a_{2}(t) X_{2}(t)= & a_{1}(t)[\Re(v) \cos [b(t+1)]-\Im(v) \operatorname{sen}[b(t+1))]+ \\
& +a_{2}(t)[\Re(v) \operatorname{sen}[b(t+1)]+\Im(v) \cos [b(t+1)]] .
\end{aligned}
$$

Assim,

$$
a_{1}(t) X_{1}(t)+a_{2}(t) X_{2}(t)=0
$$

implica,

$\left.\left[a_{1}(t) \Re(v)+a_{2}(t) \Im(v)\right] \cos [b(t+1)]+\left[a_{2}(t) \Re(v)-a_{1}(t) \Im(v)\right] \operatorname{sen}[b(t+1))\right]=0$.

Como $\cos (b t)$ e $\operatorname{sen}(b t)$ são l.i.

$$
a_{1}(t) \Re(v)+a_{2}(t) \Im(v)=0 \quad \text { e } \quad a_{2}(t) \Re(v)-a_{1}(t) \Im(v)=0 .
$$

Daí, temos dois casos a considerar:

- Se $\Re(v)$ e $\Im(v)$ forem 1.i. obtemos o resultado, ou seja, $X_{1}(t)$ e $X_{2}(t)$ são l.i.. 
- Se $\Re(v)$ e $\Im(v)$ forem l.d., consideramos ou $\Re(v)=c \Im(v), c \in \mathbb{R}$ e $\Im(v) \neq 0$, ou $\Im(v)=d \Re(v), d \in \mathbb{R}$ e $\Re(v) \neq 0$, e o resultado segue.

Lema 1.2.4. Se $w_{j}=\frac{2 j \pi}{p}, j=0,1, \ldots, p-1$, então as funçôes $e^{i w_{j} t}$ são l.i..

Prova. Sejam $a_{j}(t), j=0,1, \ldots, p-1$, funções 1-periódicas tais que

$$
\sum_{j=0}^{p-1} a_{j}(t) e^{i w_{j} t}=0, \quad \text { q.s. em } t \in \mathbb{R} \text {. }
$$

Então

$$
\sum_{j=0}^{p-1} a_{j}(t) e^{i w_{j}(t+s)}=0, \quad \text { q.s. em } t \in \mathbb{R}
$$

para $s=1, \ldots, p-1$. Isto é,

$$
\left[\begin{array}{ccccc}
1 & e^{i w_{1} t} & e^{i w_{2} t} & \ldots & e^{i w_{p-1} t} \\
1 & e^{i w_{1}(t+1)} & e^{i w_{2}(t+1)} & \ldots & e^{i w_{p-1}(t+1)} \\
1 & e^{i w_{1}(t+2)} & e^{i w_{2}(t+2)} & \ldots & e^{i w_{p-1}(t+2)} \\
\vdots & \vdots & \vdots & \ddots & \vdots \\
1 & e^{i w_{1}(t+(p-1))} & e^{i w_{2}(t+(p-1))} & \ldots & e^{i w_{p-1}(t+(p-1))}
\end{array}\right]\left(\begin{array}{c}
a_{1}(t) \\
a_{2}(t) \\
a_{3}(t) \\
\vdots \\
a_{p-1}(t)
\end{array}\right)=\left(\begin{array}{c}
0 \\
0 \\
0 \\
\vdots \\
0
\end{array}\right),
$$

q.s. em $t \in \mathbb{R}$.

Denotaremos por $B$ a matriz $p \times p$ da igualdade acima. Observe que

$$
\operatorname{det} B=e^{w_{1} i t} e^{w_{2} i t} \ldots e^{w_{p-1} i t} \operatorname{det} M=e^{i(p-1) \pi t} \operatorname{det} M \text {, }
$$

onde

$$
M=\left[\begin{array}{ccccc}
1 & 1 & 1 & \ldots & 1 \\
1 & e^{i w_{1}} & e^{i w_{2}} & \ldots & e^{i w_{p-1}} \\
1 & \left(e^{i w_{1}}\right)^{2} & \left(e^{i w_{2}}\right)^{2} & \ldots & \left(e^{i w_{p-1}}\right)^{2} \\
\vdots & \vdots & \vdots & \ddots & \vdots \\
1 & \left(e^{i w_{1}}\right)^{p-1} & \left(e^{i w_{2}}\right)^{p-1} & \ldots & \left(e^{i w_{p-1}}\right)^{p-1}
\end{array}\right]
$$


Assim, $\operatorname{det} B \neq 0$ e $a_{1}(t), a_{2}(t), \ldots, a_{p-1}(t) \equiv 0$, q.s. em $t \in \mathbb{R}$. Portanto, $e^{i w_{j} t}, j=0,1, \ldots, p-1$ são l.i..

Lema 1.2.5. Sejam $w_{j}=\frac{2 j \pi}{p}, j=0,1, \ldots, p-1$. Se $e^{i w_{j}}$ for um autovalor de $A$ com autovetor $v_{j}$, então as funções

$$
x_{j}(t)=e^{i w_{j} t} v_{j}
$$

são soluções l.i. de (1.4)

Prova. Para cada $j=0,1, \ldots, p-1$, a função $x_{j}(t)=e^{i w_{j} t} v_{j}$ é solução de (1.4). De fato,

$$
A x_{j}(t-1)=A v_{j} e^{i w_{j}(t-1)}=e^{i w_{j}} e^{i w_{j}(t-1)}=x_{j}(t)
$$

Assim, se $\sum_{j=0}^{p-1} a_{j}(t) e^{i w_{j} t} v_{j}=0$, então pelo Lema $1.2 .4, a_{0}(t), a_{1}(t), \ldots$, $a_{p-1}(t) \equiv 0$, q.s., em $t \in \mathbb{R}$.

\subsubsection{O Método de Carvalho}

O método introduzindo em [5] consiste basicamente de um resultado que caracteriza funções periódicas, com período inteiro. Enunciaremos este resultado da seguinte forma.

Dado $\xi \in \mathbb{R},[\xi]$ denota o maior inteiro menor ou igual a $\xi$.

Lema 1.2.6 (Carvalho, 1993). Se p é um inteiro positivo ex é uma função p-periódica, então

$$
x(t)=\sum_{j=0}^{\left[\frac{p}{2}\right]}\left(a_{j}(t) \cos \left(\frac{2 j \pi}{p} t\right)+b_{j}(t) \operatorname{sen}\left(\frac{2 j \pi}{p} t\right)\right),
$$

onde $a_{j}$ 's $e b_{j}$ 's são funções reais 1-periódicas, $\operatorname{com} b_{\frac{p}{2}}(t) \equiv 0$ se $p$ for par. 
Prova. A equação que define $p$-periodicidade é

$$
x(t-p)=x(t)
$$

Fazendo

$$
\begin{aligned}
x_{1}(t) & =x(t), \\
x_{2}(t) & =x_{1}(t-1), \\
x_{3}(t) & =x_{2}(t-1), \\
\vdots & \\
x_{p}(t) & =x_{p-1}(t-1),
\end{aligned}
$$

e observando que $x_{p}(t-1)=x_{1}(t)$, podemos escrever a (1.10) na seguinte forma equivalente

$$
X(t)=A X(t-1)
$$

onde

$$
X(t)=\left(\begin{array}{c}
x_{1}(t) \\
x_{2}(t) \\
x_{3}(t) \\
\vdots \\
x_{p}(t)
\end{array}\right) \quad \text { e } \quad A=\left[\begin{array}{cccccc}
0 & 0 & 0 & \ldots & 0 & 1 \\
1 & 0 & 0 & \ldots & 0 & 0 \\
0 & 1 & 0 & \ldots & 0 & 0 \\
\vdots & \vdots & \vdots & \ddots & \vdots & \vdots \\
0 & 0 & 0 & \ldots & 1 & 0
\end{array}\right] .
$$

Para esta particular matriz $A$, um simples exame da equação $\operatorname{det}(A-\lambda I)=0$ nos leva à conclusão de que os autovalores de $A$ são as raízes $p$-ésimas da unidade. Assim,

$$
\lambda_{j}=\cos w_{j}+i \operatorname{sen} w_{j}, \quad w_{j}=\frac{2 j \pi}{p}, j=0,1, \ldots, p-1,
$$

isto é:

- Se $p$ for par,

$$
\lambda_{0}=1, \lambda_{\frac{p}{2}}=-1, \lambda_{j}=\cos w_{j}+i \operatorname{sen} w_{j}, \text { e } \bar{\lambda}_{j}, j=1, \ldots, \frac{p-2}{2} .
$$


- Se $p$ for ímpar,

$$
\lambda_{0}=1, \lambda_{j}=\cos w_{j}+i \operatorname{sen} w_{j}, \text { e } \bar{\lambda}_{j}, j=1, \ldots, \frac{p-1}{2} .
$$

Primeiro vamos supor $p$ par e considerar $v_{0}, v_{1}, \ldots, v_{p / 2}, \bar{v}_{1}, \ldots, \bar{v}_{(p-2) / 2}$, como sendo os autovetores associados aos autovalores $\lambda_{0}, \lambda_{1}, \ldots, \lambda_{p / 2}, \bar{\lambda}_{1}, \ldots, \bar{\lambda}_{(p-2) / 2}$, respectivamente.

Precisamos apenas dos autovetores $v_{0}, v_{1}, \ldots, v_{(p-2) / 2}, v_{p / 2}$. Pelo Lema 1.2.3

$$
\begin{gathered}
X_{1}^{j}(t)=\Re\left(v_{j}\right) \cos \left[w_{j}(t+1)\right]-\Im\left(v_{j}\right) \operatorname{sen}\left[w_{j}(t+1)\right], \\
X_{2}^{j}(t)=\Re\left(v_{j}\right) \operatorname{sen}\left[w_{j}(t+1)\right]+\Im\left(v_{j}\right) \cos \left[w_{j}(t+1)\right], \quad j=0,1, \ldots, \frac{p}{2},
\end{gathered}
$$

são soluções de (1.11).

Como $w_{0}=0, w_{\frac{2}{2}}=\pi$ e $A v_{0}=v_{0}$, obtemos que $\Re\left(v_{0}\right)$ e $\Im\left(v_{0}\right)$ são l.d., daí $X_{1}^{0}$ e $X_{2}^{0}$ são l.d.. Analogamente, $X_{1}^{\frac{p}{2}}$ e $X_{2}^{\frac{p}{2}}$ são l.d.. Mas, para $j=1, \ldots, \frac{p-2}{2}, X_{1}^{j}$ e $X_{2}^{j}$ são l.i...

Para provar que $X_{1}^{0}, X_{1}^{\frac{p}{2}}, X_{1}^{j}$ e $X_{2}^{j}, j=1, \ldots, \frac{p-2}{2}$, são l.i., basta mostrar que as funções

$$
1, \cos (\pi t), \cos \left(w_{j} t\right), \operatorname{sen}\left(w_{j} t\right), \quad j=1, \ldots, \frac{p-2}{2}
$$

são l.i...

De fato,

$$
\sum_{j=0}^{\frac{p}{2}} a_{j}(t) X_{1}^{j}+b_{j}(t) X_{2}^{j}=\sum_{j=0}^{\frac{p}{2}}\left[\alpha_{j}(t) \cos \left(w_{j} t\right)+\beta_{j}(t) \operatorname{sen}\left(w_{j} t\right)\right]
$$

onde

$$
\begin{aligned}
& \alpha_{j}(t):=a_{j}(t)\left[\Re\left(v_{j}\right) \cos w_{j}-\Im\left(v_{j}\right) \operatorname{sen} w_{j}\right]+b_{j}(t)\left[\Re\left(v_{j}\right) \operatorname{sen} w_{j}+\Im\left(v_{j}\right) \cos w_{j}\right] \\
& \beta_{j}(t):=-a_{j}(t)\left[\Re\left(v_{j}\right) \operatorname{sen} w_{j}+\Im\left(v_{j}\right) \cos w_{j}\right]+b_{j}(t)\left[\Re\left(v_{j}\right) \cos w_{j}+\Im\left(v_{j}\right) \operatorname{sen} w_{j}\right]
\end{aligned}
$$


Observe que o $p / 2$-ésimo termo dessa soma é

$$
\alpha_{\frac{p}{2}}(t) \cos (\pi t)+\beta_{\frac{p}{2}}(t) \operatorname{sen}(\pi t)
$$

$\operatorname{como} \operatorname{sen}(\pi t)=\tan (\pi t) \cos (\pi t)$, definimos

$$
\hat{\alpha}_{\frac{p}{2}}(t):=\alpha_{\frac{p}{2}}(t)+\beta_{\frac{p}{2}}(t) \tan (\pi t)
$$

para obter

$$
\begin{aligned}
& \sum_{j=0}^{\frac{p}{2}}\left[\alpha_{j}(t) \cos \left(w_{j} t\right)+\beta_{j}(t) \operatorname{sen}\left(w_{j} t\right)\right] \\
& =\alpha_{0}(t)+\hat{\alpha}_{\frac{p}{2}}(t) \cos (\pi t)+\sum_{j=1}^{\frac{p-2}{2}}\left(\alpha_{j}(t) \cos \left(w_{j} t\right)+\beta_{j}(t) \operatorname{sen}\left(w_{j} t\right)\right) \\
& =\alpha_{0}(t)+\hat{\alpha}_{\frac{p}{2}}(t) \cos (\pi t)+\sum_{j=1}^{\frac{p-2}{2}}\left(\alpha_{j}(t) \frac{e^{i w_{j} t}+e^{-i w_{j} t}}{2}+\beta_{j}(t) \frac{e^{i w_{j} t}-e^{-i w_{j} t}}{2 i}\right) \\
& =\alpha_{0}(t)+\hat{\alpha}_{\frac{p}{2}}(t) \cos (\pi t)+\sum_{j=1}^{\frac{p-2}{2}}\left(\frac{\alpha_{j}(t)-i \beta_{j}(t)}{2} e^{i w_{j} t}+\frac{\alpha_{j}(t)+i \beta_{j}(t)}{2} e^{-i w_{j} t}\right) \\
& =\alpha_{0}(t)+\hat{\alpha}_{\frac{p}{2}}(t) \cos (\pi t)+\sum_{j=1}^{\frac{p-2}{2}}\left(\frac{\alpha_{j}(t)-i \beta_{j}(t)}{2} \lambda_{j}^{t}+\frac{\alpha_{j}(t)+i \beta_{j}(t)}{2} \bar{\lambda}_{j}^{t}\right) .
\end{aligned}
$$

Definindo $\alpha_{j}:=\alpha_{j-p / 2}$ e $\beta_{j}:=\beta_{j-p / 2}$, para $j=(p+2) / 2, \ldots, p-1$, obtemos

$$
\begin{aligned}
& \sum_{j=0}^{\frac{p}{2}}\left[\alpha_{j}(t) \cos \left(w_{j} t\right)+\beta_{j}(t) \operatorname{sen}\left(w_{j} t\right)\right]= \\
& =\alpha_{0}(t)+\hat{\alpha}_{\frac{p}{2}}(t) \cos (\pi t)+\sum_{j=1}^{\frac{p-2}{2}} \frac{\alpha_{j}(t)-i \beta_{j}(t)}{2} \lambda_{j}^{t}+\sum_{j=\frac{p+2}{2}}^{p-1} \frac{\alpha_{j}(t)+i \beta_{j}(t)}{2} \lambda_{j}^{t},
\end{aligned}
$$

isto é,

$$
\sum_{j=0}^{\frac{p}{2}}\left[\alpha_{j}(t) \cos \left(w_{j} t\right)+\beta_{j}(t) \operatorname{sen}\left(w_{j} t\right)\right]=\sum_{j=0}^{p-1} c_{j}(t) \lambda_{j}^{t}
$$


onde

$$
c_{j}(t)= \begin{cases}\frac{\alpha_{0}(t),}{\frac{\alpha_{j}(t)-i \beta_{j}(t)}{2},}, & \text { se } 1 \leq j \leq \frac{p}{2}-1, \\ \hat{\alpha}_{j}, & \text { se } j=\frac{p}{2}, \\ \frac{\alpha_{j}(t)+i \beta_{j}(t)}{2}, & \text { se } \frac{p}{2}+1 \leq j \leq p-1,\end{cases}
$$

com $\alpha_{j}$ 's, $\beta_{j}$ 's, funções 1-periódicas definidas em (1.12).

Pelo Lema $1.2 .5, c_{j}(t)=0$, q.s. em $t$, para todo $j$. E concluímos que $\alpha_{0}(t)$, $\hat{\alpha}_{\frac{p}{2}}(t), \alpha_{j}(t), \beta_{j}(t)$ são nulas q.s. em $t$.

Pelo Lema 1.2.2, qualquer outra solução de (1.11) é escrita de forma única como combinação dessas soluções l.i., isto é, existem

$$
\tilde{a}_{0}(t), \tilde{a}_{\frac{p}{2}}(t), \tilde{a}_{j}(t), \tilde{b}_{j}(t) \quad j=1, \ldots, \frac{p-2}{2},
$$

1-periódicas tais que

$$
\begin{aligned}
X(t)= & \tilde{a}_{0}(t) \Re\left(v_{0}\right)+\tilde{a}_{\frac{p}{2}}(t)\left(\Re\left(v_{\frac{p}{2}}\right) \cos [\pi(t+1)]-\Im\left(v_{\frac{p}{2}}\right) \operatorname{sen}[\pi(t+1)]\right) \\
& +\sum_{j=1}^{\frac{p-2}{2}} \tilde{a}_{j}(t)\left(\Re\left(v_{j}\right) \cos \left[w_{j}(t+1)\right]-\Im\left(v_{j}\right) \operatorname{sen}\left[w_{j}(t+1)\right]\right) \\
& +\sum_{j=1}^{\frac{p-2}{2}} \tilde{b}_{j}(t)\left(\Re\left(v_{j}\right) \operatorname{sen}\left[w_{j}(t+1)\right]+\Im\left(v_{j}\right) \cos \left[w_{j}(t+1)\right]\right) .
\end{aligned}
$$

Fazendo

$$
\begin{aligned}
\tilde{a}_{0}(t) & :=\tilde{a}_{0}(t) \Re\left(v_{0}\right), \\
\tilde{\tilde{a}}_{\frac{p}{2}}(t) & :=\tilde{a}_{\frac{p}{2}}(t)\left[-\Re\left(v_{\frac{p}{2}}\right)+\Im\left(v_{\frac{p}{2}}\right) \tan (\pi t)\right], \\
\tilde{\tilde{a}}_{j}(t) & :=\tilde{a}_{j}(t)\left[\Re\left(v_{j}\right) \cos w_{j}-\Im\left(v_{j}\right) \operatorname{sen} w_{j}\right]+\tilde{b}_{j}(t)\left[\Re\left(v_{j}\right) \operatorname{sen} w_{j}+\Im\left(v_{j}\right) \cos w_{j}\right], \\
\tilde{\tilde{b}}_{j}(t) & :=-\tilde{a}_{j}(t)\left[\Re\left(v_{j}\right) \operatorname{sen} w_{j}+\Im\left(v_{j}\right) \cos w_{j}\right]+\tilde{b}_{j}(t)\left[\Re\left(v_{j}\right) \cos w_{j}-\Im\left(v_{j}\right) \operatorname{sen} w_{j}\right],
\end{aligned}
$$

obtemos

$$
X(t)=\tilde{\tilde{a}}_{0}(t)+\tilde{\tilde{a}}_{\frac{p}{2}}(t) \cos (\pi t)+\sum_{j=1}^{\frac{p-2}{2}}\left(\tilde{\tilde{a}}_{j}(t) \cos \left(w_{j} t\right)+\tilde{\tilde{b}}_{j}(t) \operatorname{sen}\left(w_{j} t\right)\right) .
$$


Observe que $\tilde{\tilde{a}}_{j}$ 's e $\tilde{\tilde{b}}_{j}$ 's são 1-periódicas.

De (1.11), temos que

$$
x(t)=a_{0}(t)+a_{\frac{p}{2}}(t) \cos (\pi t)+\sum_{j=1}^{\frac{p-2}{2}}\left(a_{j}(t) \cos \left(w_{j} t\right)+b_{j}(t) \operatorname{sen}\left(w_{j} t\right)\right)
$$

onde $a_{j}$ 's e $b_{j}$ 's são as primeiras coordenadas dos vetores $\tilde{\tilde{a}}_{j}$ 's e $\tilde{\tilde{b}}_{j}$ 's, respectivamente.

Para $p$ ímpar, o raciocínio é análogo.

Condições necessárias e suficientes para que uma equação do tipo (3) possua solução $p$-periódica, $p>0$ inteiro, são obtidas da seguinte forma:

Seja $p$ um inteiro positivo e considere uma função $x$ de classe $C^{1}, p$-periódica. Pelo Lema 1.2.6 existem funções $a_{j}, b_{j} 1$-periódicas, tais que

$$
x(t)=\sum_{j=0}^{\left[\frac{p}{2}\right]}\left[a_{j}(t) \cos \left(w_{j} t\right)+b_{j}(t) \operatorname{sen}\left(w_{j} t\right)\right]
$$

e

$$
\dot{x}(t)=\sum_{j=0}^{\left[\frac{p}{2}\right]}\left[\left[\dot{a}_{j}(t)+w_{j} b_{j}(t)\right] \cos \left(w_{j} t\right)+\left[\dot{b}_{j}(t)-w_{j} a_{j}(t)\right] \operatorname{sen}\left(w_{j} t\right)\right]
$$

com $b_{\frac{p}{2}}(t) \equiv 0$ se $p$ for par.

Se, além disso, $x$ é uma solução de (3), então $f\left(\alpha, t, x_{t}\right)$ é uma função $p$ periódica, assim existem funções 1-periódicas, $u_{j}(t), v_{j}(t)$, que dependem de $a_{j}(t)$, $b_{j}(t)$ e de $\alpha$, tais que

$$
f\left(\alpha, t, x_{t}\right)=\sum_{j=0}^{\left[\frac{p}{2}\right]}\left[u_{j}(t) \cos \left(w_{j} t\right)+v_{j}(t) \operatorname{sen}\left(w_{j} t\right)\right]
$$

com $v_{\frac{p}{2}}(t) \equiv 0$ se $p$ for par.

Daí, usando a independência linear de

$$
\left\{1, \cos \left(w_{j} t\right), \operatorname{sen}\left(w_{j} t\right), j=1, \ldots,[p / 2]\right\}
$$


temos um sistema de equações diferenciais ordinárias, que depende do campo vetorial $f$, isto é, dos coeficientes $u_{j}$ e $v_{j}$. Logo, este sistema pode ser escrito como:

$$
\begin{aligned}
\dot{a}_{0}(t) & =u_{0}(t), \\
{\left[a_{\frac{p}{2}}(t) \cos (\pi t)\right] } & =u_{\frac{p}{2}}(t) \cos (\pi t), \\
\dot{a}_{j}(t) & =u_{j}(t)-w_{j} b_{j}(t), \\
\dot{b}_{j}(t) & =v_{j}(t)+w_{j} a_{j}(t), \quad j=1, \ldots,\left[\frac{p-1}{2}\right],
\end{aligned}
$$

se $p$ for par ou

$$
\begin{aligned}
& \dot{a}_{0}(t)=u_{0}(t) \\
& \dot{a}_{j}(t)=u_{j}(t)-w_{j} b_{j}(t), \\
& \dot{b}_{j}(t)=v_{j}(t)+w_{j} a_{j}(t), \quad j=1, \ldots,\left[\frac{p-1}{2}\right],
\end{aligned}
$$

se $p$ for ímpar. Ficando estabelecido o seguinte resultado:

Teorema 1.2.1. Seja $p$ um inteiro positivo. A Equação (3) tem solução pperiódica se, e somente se, existir uma solução 1-periódica para o Sistema (1.17), se $p$ for par, ou para o Sistema (1.18), se p for ímpar.

\subsection{Estabilidade}

A solução trivial, $x(t) \equiv 0$, de (2) é estável se para cada $\epsilon>0$ existir $\delta=\delta(\epsilon)>0$ tal que, para qualquer $\varphi \in C([-\tau, 0], \mathbb{R}) \operatorname{com}\|\varphi\|<\delta$, a solução $x=x(t ; \varphi)$ de (2) satisfaz

$$
|x(t)|<\epsilon, \quad \text { para todo } t \geq-\tau \text {. }
$$

Caso contrário, a solução trivial de (2) é chamada instável. A solução trivial de (2) é assintoticamente estável se for estável e existir $\delta_{0}>0$ tal que, para qualquer 
$\varphi \in C([-\tau, 0], \mathbb{R})$ com $\|\varphi\|<\delta_{0}$, a solução $x(t):=x(t ; \varphi)$ de $(2)$ satisfaz

$$
\lim _{t \rightarrow \infty} x(t)=0 .
$$

A estabilidade da solução trivial de (2) depende dos coeficientes $a_{j}$ 's e dos retardamentos $r_{j}$ 's e pode ser determinada localizando, no plano complexo, as raízes da equação característica associada

$$
\Delta(\lambda)=\lambda-\sum_{j=0}^{l} a_{j} e^{-\lambda r_{j}}=0, \quad r_{0}=0 .
$$

Quando for importante salientar a dependência dos parâmetros $a_{0}, \ldots, a_{l}$ usaremos a notação $\Delta(\lambda)=\Delta\left(a_{0}, \ldots, a_{l} ; \lambda\right)$.

O teorema seguinte é uma versão particular, conveniente aos nossos propósitos, de um resultado apresentado em Dieudonné [13] (Teorema 9.17.3) como aplicação do Teorema de Rouché. Estabelece a continuidade das raízes de uma equação transcendente em $\mathbb{C}$ em relação aos parâmetros. Este resultado será muito útil no estudo de estabilidade que faremos mais adiante.

Teorema 1.3.1. Sejam $\Omega$ um conjunto aberto em $\mathbb{C}$ e $F$ uma função contínua a valores complexos definida em $\mathbb{R}^{l+1} \times \Omega$ tal que, para cada $\left(a_{0}, \ldots, a_{l}\right) \in \mathbb{R}^{l+1}$,

$$
z \mapsto F\left(a_{0}, \ldots, a_{l} ; z\right)
$$

é analítica em $\Omega$. Seja $W$ um subconjunto aberto de $\Omega$ cujo fecho $\bar{W}$ em $\mathbb{C}$ é compacto e contido em $\Omega$. Seja $\left(a_{0}^{0}, \ldots, a_{l}^{0}\right)$ tal que $F\left(a_{0}^{0}, \ldots, a_{l}^{\mathbf{0}} ; z\right)$ não tem zeros na fronteira de $W$. Então, existe uma vizinhança $U$ de $\left(a_{0}^{0}, \ldots, a_{l}^{0}\right)$ em $\mathbb{R}^{l+1}$ tal que

(i) para qualquer $\left(a_{0}, \ldots, a_{l}\right) \in U$, a equação $F\left(a_{0}, \ldots, a_{l} ; z\right)=0$ não tem raízes na fronteira de $W$;

(ii) a quantidade de raízes da equação $F\left(a_{0}, \ldots, a_{l} ; z\right)=0$ em $W$, levando em conta suas multiplicidades, é constante para $\left(a_{0}, \ldots, a_{l}\right) \in U$. 

A função

$$
\left(a_{0}, \ldots, a_{l} ; \lambda\right) \mapsto \Delta\left(a_{0}, \ldots, a_{l} ; \lambda\right)
$$

de $\mathbb{R}^{l+1} \times \mathbb{C}$ dada em (1.19) satisfaz as condições impostas à função $F$ no Teorema 1.3.1.

\subsubsection{Equações Diferenciais a Diferenças}

Os resultados que apresentaremos a seguir são ferramentas muito úteis para tratar da estabilidade assintótica da solução trivial de (2). O teorema abaixo é bem conhecido.

Teorema 1.3.2. Se $\Re(\lambda)<0$ para toda raiz da equação característica (1.19), então existem constantes positivas $M$ e $\gamma$ tais que

$$
|x(t)| \leq M\|\varphi\| e^{-\gamma\left(t-t_{0}\right)}, \quad t \geq t_{0} .
$$

O resultado seguinte pode ser encontrado em [12].

Teorema 1.3.3. Seja $x$ uma solução de (1.4) correspondente a alguma função inicial $\varphi$. Para qualquer $\gamma \in \mathbb{R}$ tal que $\Delta(\lambda) \neq 0$ na reta $\Re(\lambda)=\gamma$, temos $a$ expansão assintótica

$$
x(t)=\sum_{j=1}^{l} p_{j}(t) e^{\lambda_{j} t}+o\left(e^{\gamma t}\right) \quad \text { para } t \rightarrow \infty,
$$

onde $\lambda_{1}, \ldots, \lambda_{l}$ é a quantidade finita de zeros de $\Delta(\lambda)$ tais que $\Re\left(\lambda_{j}\right)>\gamma, j=$ $1, \ldots, l$ e $p_{j}(t)$ é um polinômio de grau menor ou igual a $m_{j}-1$, onde $m_{j}$ é a multiplicidade de $\lambda_{j}$ como um zero de $\Delta(\lambda)$.

Corolário 1.3.1. Se $\Delta(\lambda)$ não tem zeros no semi-plano $\{\lambda: \Re(\lambda) \geq 0\}$, todas as soluções de (1.4) convergem para zero exponencialmente quando $t \rightarrow \infty$. 
Assim, (2) é assintoticamente estável se qualquer raiz da equação (1.19) tem parte real negativa. Por outro lado, se uma raiz de (1.19) tem parte real positiva, (2) é instável.

A seguinte proposição está enunciada apenas para o caso de dois retardamentos por Hale e Huang [18].

Proposiçāo 1.3.1. Se $\sum_{i=0}^{l} a_{i}>0$, então (2) é instável para todos os valores dos retandamentos $r_{i}, i=0, \ldots, l$.

Prova. De fato, basta observar que $\Delta(\lambda)$ é uma função analítica em $\lambda$,

$$
\Delta(0)=-\sum_{i=0}^{l} a_{i}<0
$$

$\Delta(\lambda) \in \mathbb{R}$, se $\lambda \in \mathbb{R}$ e $\lim _{\lambda \rightarrow \infty} \Delta(\lambda)=\infty$. Logo, existe uma raiz real positiva.

Definiçāo 1.3.1. A região de estabilidade de (2) é um subconjunto, $D$, do espaço dos parâmetros $a_{j}, j=0, \ldots, l$, tal que (2) é assintoticamente estável se, $e$ somente se, $\left(a_{0}, \ldots, a_{i}\right) \in D$.

O conhecimento da fronteira da região de estabilidade assintótica é importante para a caracterização das regiões onde existem soluções periódicas.

Várias técnicas têm sido elaboradas para determinar as regiões de estabilidade de equações do tipo (1.1'9).

A equação

$$
\varepsilon \dot{x}(t)=a_{0} x(t)+a_{1} x\left(t-r_{1}\right)
$$

é um caso particular de (1.19). Se $\varepsilon>0$ e $r_{1}>0$, por uma conveniente re-escala no tempo $\left(t \leftrightarrow t / r_{1}\right)$ e uma mudança de parâmetros, a equação acima se reduz à forma

$$
\dot{x}(t)=a x(t)+b x(t-1) .
$$


Há bastante tempo, o problema de estabilidade para (1.20) foi completamente resolvido por Hayes [19] que estabeleceu o seguinte resultado.

Teorema 1.3.4 (Hayes, 1950). As raizes da equação $(\lambda-a) e^{\lambda}-b=0$, onde $a$ e b são reais, têm partes reais negativas se, e somente se,

$$
\left\{\begin{array}{l}
a<1 \\
a+b<0 \\
b>\zeta \operatorname{sen} \zeta+a \cos \zeta
\end{array}\right.
$$

onde $\zeta$ é $a$ raiz de $\zeta=a \tan \zeta, 0<\zeta<\pi$, se $a \neq 0$ e $\zeta=\pi / 2$ se $a=0$.

Hale e Huang [18] em 1993, consideraram o estudo de estabilidade de

$$
\dot{x}(t)=-a x(t)-b x(t-r)-c x(t-\sigma),
$$

no plano $r \sigma$ para vários valores fixados de $a, b, c$. Num contexto de aplicações, Mizumo e Ikeda [27], 1989, observaram propriedades de estabilidade de

$$
\gamma^{-1} \dot{\phi}(t)=-\phi(t)+\eta A\left[\phi\left(t-t_{1}\right)+\phi\left(t-t_{2}\right)\right],
$$

para explicar alguns resultados de experimentos com laser. A equação (1.22) é uma versão de (1.21) com os parâmetros vinculados pelas relações $a=\gamma>0 \mathrm{e}$ $b=c=-\eta \gamma A$.

Normalizando os retardamentos pela mesma re-escala no tempo utilizada para obter (1.20) e assumindo $r>\sigma$ (o que não representa perda de generalidade), (1.21) se reduz à forma

$$
\dot{x}(t)=-A x(t)-B x(t-1)-C x(t-R), \quad 0 \leq R<1,
$$

onde $A, B, C$ são novos parâmetros e $R=\sigma / r$. Recentemente, Mahaffy et al. [25] examinaram a estabilidade no espaço dos parâmetros $(A, B, C)$ para diferentes valores da razão dos retardamentos, isto é, $0 \leq R \leq 1 / 2$. 
Philos [29], considerou a equação diferencial retardada

$$
\dot{x}(t)=a x(t)+\sum_{j=1}^{k} b_{j} x\left(t-\tau_{j}\right),
$$

onde $k$ é um inteiro positivo, $a$ e $b_{j}, j=1, \ldots, k$, são constantes reais não nulas, e $\tau_{j}, j=1, \ldots, k$, são números reais positivos dois a dois distintos. Inspirado em resultados parciais de [14], ele estuda propriedades de (1.23) sem, entretanto, caracterizar as regiões de estabilidade no espaço dos parâmetros $a, b_{j}, j=1, \ldots, k$.

\subsubsection{Equações a Diferenças}

Os resultados apresentados aqui também serão úteis no estudo que faremos no Capítulo 4.

Teorema 1.3.5 (Levin e May, 1976). Se $q \in \mathbb{R}$ ek $k\{0,1,2, \ldots\}$, então a equação a diferença

$$
x_{n+1}-x_{n}-q x_{n-k}=0,
$$

é assintoticamente estável se, e somente se

$$
0<q<2 \cos \left(\frac{k \pi}{2 k+1}\right) .
$$

Teorema 1.3.6 (Clark, 1976). Sejam $p, q \in \mathbb{R} e k \in\{0,1,2, \ldots\}$. Então,

$$
|p|+|q|<1
$$

é uma condição suficiente para a estabilidade assintótica da equação a diferença

$$
x_{n+1}+p x_{n}+q x_{n-k}=0 .
$$

Utilizando os mesmos argumentos de Clark [9], obtemos o seguinte resultado. 
Teorema 1.3.7. Suponha que $\left(\alpha_{1}, \ldots, \alpha_{l}\right) \in \mathbb{R}^{l}$. Então,

$$
\sum_{r=1}^{l}\left|\alpha_{r}\right|<1
$$

é uma condição suficiente para a estabilidade assintótica da equação a diferença

$$
x(t)=\sum_{r=1}^{l} \alpha_{r} x(t-r) .
$$

Prova. A equação característica é

$$
e^{\lambda l}=\sum_{r=1}^{l} \alpha_{r} e^{\lambda(l-r)}
$$

ou definindo $\mu:=e^{\lambda}$, temos

$$
\mu^{l}=\sum_{r=1}^{l} \alpha_{r} \mu^{l-r} .
$$

Sejam $f(\mu)=\mu^{l}$ e $g(\mu)=\sum_{r=1}^{l} \alpha_{r} \mu^{l-r}$. Observe que, se $|\mu|=1$ temos

$$
|g(\mu)| \leq \sum_{r=1}^{l}\left|\alpha_{r}\right|<1=|f(\mu)| .
$$

Assim, pelo Teorema de Rouché, $f(\mu)$ e $f(\mu)-g(\mu)$ têm exatamente $l$ zeros no disco unitário. Portanto, a solução $x(t) \equiv 0$ é assintoticamente estável.

Os dois resultados seguintes, devidos a Carvalho [5], caracterizam as regiões de estabilidade assintótica absoluta (isto é, estabilidade assintótica para todos os retardamentos) e instabilidade absoluta, no plano dos parâmetros $a, b$, para a equação

$$
x(t)=a x(t-r)+b x(t-s),
$$

onde $a, b, r$ e $s$ são constantes reais. Estes resultados são obtidos analisando a equação característica associada

$$
1=a e^{-\lambda r}+b e^{-\lambda s} .
$$


Teorema 1.3.8. Qualquer raiz da equação (1.27) tem parte real negativa para qualquer par de retardamentos $0<r, s<\infty$ se, e somente se,

$$
|a|+|b|<1 \text {. }
$$

Se $|a|+|b|=1$ e $a<0$ ou $b<0$, então existe somente uma categoria de retardamentos entre as 3 seguintes:

$$
\begin{aligned}
& C_{1}=\left\{(r, s) \in\left(\mathbb{R}_{+}^{*}\right)^{2}: \frac{r}{s}=\frac{\text { impar }}{\text { impar }}\right\}, \\
& C_{2}=\left\{(r, s) \in\left(\mathbb{R}_{+}^{*}\right)^{2}: \frac{r}{s}=\frac{\text { impar }}{\text { par }}\right\}, \\
& C_{3}=\left\{(r, s) \in\left(\mathbb{R}_{+}^{*}\right)^{2}: \frac{r}{s}=\frac{\text { par }}{\text { impar }}\right\}, \\
& C_{4}=\left\{(r, s) \in\left(\mathbb{R}_{+}^{*}\right)^{2}: \frac{r}{s}=\text { irracional }\right\}
\end{aligned}
$$

que produz uma raiz para (1.27) com parte real nula; esta classe é determinada de modo único pelo sinal de a e b e, além disso, as outras três categorias restantes produzem somente raízes com parte real negativa.

Aqui, $\mathbb{R}_{+}^{*}$ denota o conjunto dos números reais positivos.

Teorema 1.3.9. Qualquer raiz da equação (1.27) tem parte real positiva para $(a, b, r, s) \in A_{i} \times B_{i}, i=1,2$, onde

$$
\begin{aligned}
& A_{1}=\left\{(a, b) \in \mathbb{R}^{2}:|b|>|a|+1\right\}, \\
& A_{2}=\left\{(a, b) \in \mathbb{R}^{2}:|a|>|b|+1\right\}, \\
& B_{1}=\left\{(r, s) \in \mathbb{R}^{2}: 0<r \leq s<\infty\right\}, \\
& B_{2}=\left\{(r, s) \in \mathbb{R}^{2}: 0<s \leq r<\infty\right\} .
\end{aligned}
$$


Sabemos que a região de estabilidade pode ser ampliada dependendo dos retardamentos. O Teorema 1.3.8 nos garante que a região mínima de estabilidade é - quadrado $|a|+|b|<1$ e, dependendo dos retardamentos, ela pode ser ampliada fora das regiões $A_{1}$ e $A_{2}$ do Teorema 1.3.9. Veja a figura a seguir.

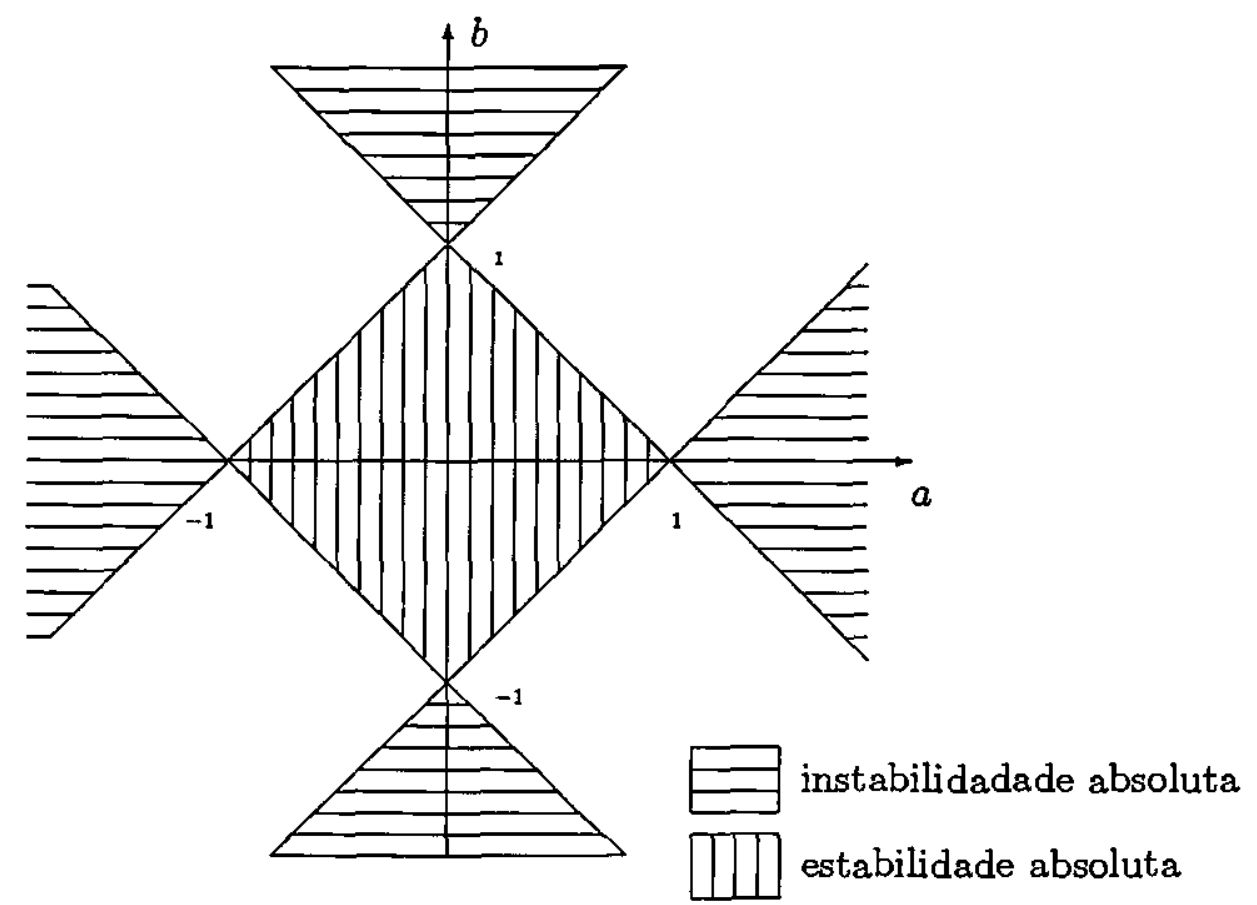

Figura 1.1: Regiões de estabilidade e instabilidade absoluta para (1.26).

Teorema 1.3.10 (Kuruklis, 1994). Sejam $a \leq 0$ e $b$ números reais, $k$ um inteiro positivo. A equação

$$
x_{n+1}-a x_{n}+b x_{n-k}=0, \quad n=0,1,2, \ldots
$$

é assintoticamente estável se, e somente se,

$$
\begin{gathered}
|a|<\frac{k+1}{k}, e \\
|a|-1<b<\left(a^{2}+1-2+|a| \cos \phi\right)^{\frac{1}{2}} \text { para } k \text { ímpar, }
\end{gathered}
$$




$$
|b-a|<1 \text { e }|b|<\left(a^{2}+1-2|a| \cos \phi\right)^{\frac{1}{2}} \text { para } k \text { par }
$$

onde $\phi$ é a solução em $(0, \pi /(k+1)) d e|a| \operatorname{sen}(k \theta)=\operatorname{sen}((k+1) \theta)$.

Estas regiões podem ser visualizadas nas figuras abaixo.
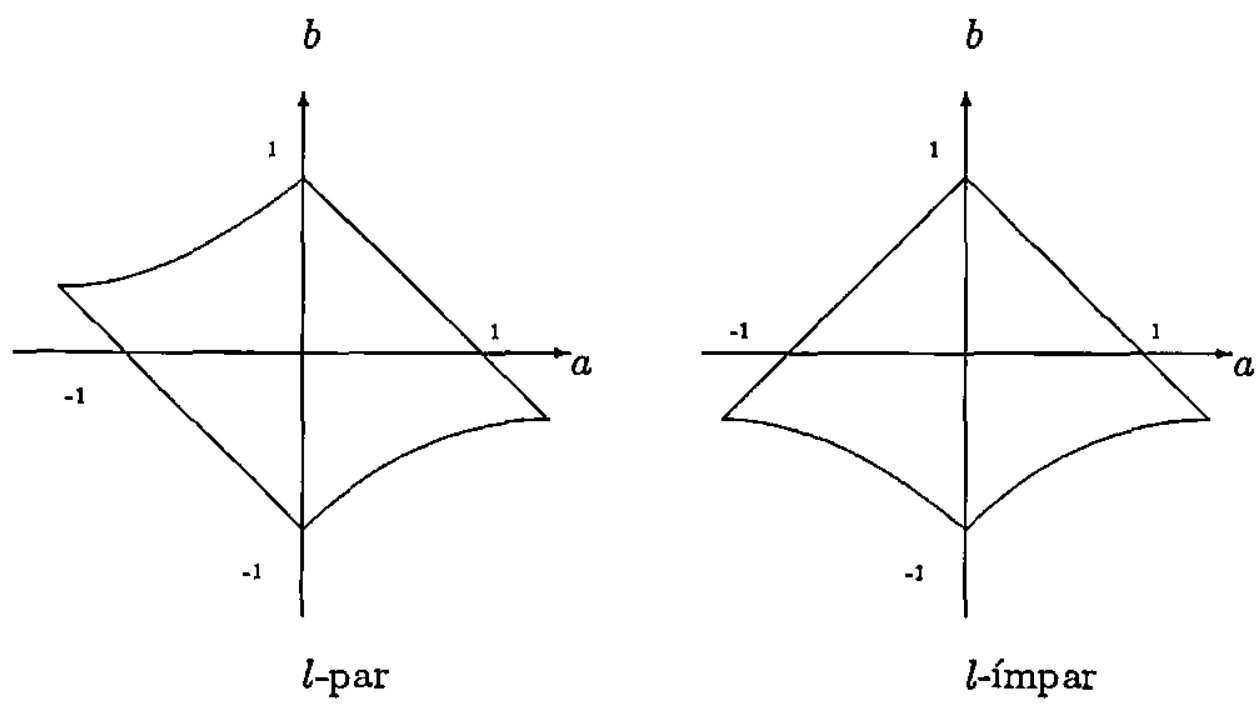

Figura 1.2:

Comparando com a Figura 1.1 notamos que as partes das regiões descritas na Figura 1.2 que não estão contidas no quadrado $(1,0)(0,1)(-1,0)(0,-1)$, estão contidas necessariamente na regiões $A_{1}, A_{2}$ da Figura 1.1 


\section{Capítulo 2}

\section{Periodicidade em um Problema}

\section{de Perturbação Singular com Vários Retardamentos}

\subsection{O Problema}

Vamos supor que os retardamentos $r_{j}$ 's de (1) são racionais, então existem inteiros $m_{j}$ 's e $m$ tais que $r_{j}=\frac{m_{j}}{m}, j=1, \ldots, \kappa$. Fazendo $x(t)=y\left(\frac{t}{m}\right)$ e $G=\frac{1}{m} F$, obtemos

$$
\dot{x}(t)=G\left(x(t), x\left(t-m_{1}\right), \ldots, x\left(t-m_{\kappa}\right)\right) .
$$

Este fato mostra ser natural estudar equações com retardamentos inteiros.

Estamos particularmente interessados no seguinte problema de perturbação singular:

$$
\varepsilon \dot{x}(t)=G\left(x(t), x\left(t-m_{1}\right), \ldots, x\left(t-m_{\kappa}\right)\right), \quad \varepsilon>0 .
$$

Mais precisamente, queremos estabelecer relações entre (2.2) e seu limite formal para $\varepsilon \rightarrow 0$ :

$$
G\left(x(t), x\left(t-m_{1}\right), \ldots, x\left(t-m_{\kappa}\right)\right)=0 .
$$


Como $G(0, \ldots, 0)=0$ e $G$ é diferenciável então, (2.2) pode ser escrita na forma

$$
\varepsilon \dot{x}(t)=\alpha_{0} x(t)+\sum_{j=1}^{\kappa} \alpha_{j} x\left(t-m_{j}\right)+h\left(x(t), x\left(t-m_{1}\right), \ldots, x\left(t-m_{\kappa}\right)\right)
$$

onde $\alpha_{j}=D_{j} G(0, \ldots, 0)$ é a derivada na origem de $G$ com relação à $j$-ésima variável, $j=Q, \ldots, \kappa$, e se $\xi=\left(x_{0}, \ldots, x_{k+1}\right), h(\xi)=o(|\xi|)$, com $|\xi| \rightarrow 0$.

Vamos estudar a parte linear de (2.4)

$$
\varepsilon \dot{x}(t)=\alpha_{0} x(t)+\sum_{j=1}^{\kappa} \alpha_{j} x\left(t-m_{j}\right)
$$

Não há perda de generalidade em supor que $m_{1}<m_{2}<\cdots<m_{\kappa}$ e redefinindo os coeficientes $\alpha_{j}, 0 \leq j \leq l:=m_{\kappa}$, se necessário, podemos escrever (2.5) na forma

$$
\varepsilon \dot{x}(t)=\sum_{r=0}^{l} \alpha_{r} x(t-r) .
$$

Nos últimos anos, o problema de existência de soluções periódicas tem sido objeto de grande interesse de pesquisa para alguns casos particulares de (2.6).

É conhecido - veja por exemplo, Hale [17] e referências ali contidas - que (2.6) $\operatorname{com} \varepsilon=1, l=1, \alpha_{0}=0$ e $\alpha_{1}=-\pi / 2$, isto é,

$$
\dot{x}(t)=-\frac{\pi}{2} x(t-1)
$$

tem soluções 4-periódicas da forma

$$
x(t)=c_{1} \operatorname{sen}\left(\frac{\pi}{2} t\right)+c_{2} \cos \left(\frac{\pi}{2} t\right), \quad c_{1}, c_{2} \in \mathbb{R} .
$$

Carvalho generalizou este fato em [5], mostrando que (2.6) $\operatorname{com} \varepsilon=1, l=$ $1, \alpha_{0}=0$ e $\alpha_{1}=(4 n-1) \pi / 2, n \in \mathbb{Z}$, isto é,

$$
\dot{x}(t)=(4 n-1) \frac{\pi}{2} x(t-1),
$$

tem soluções 4-periódicas da forma

$$
x(t)=c_{1} \operatorname{sen}\left((4 n-1) \frac{\pi}{2} t\right)+c_{2} \cos \left((4 n-1) \frac{\pi}{2} t\right), \quad c_{1}, c_{2} \in \mathbb{R} .
$$


Utilizando um método desenvolvido por Carvalho [5], descrito anteriormente no Cap. 1, estudamos a localização das $(l+1)$-uplas $\left(\alpha_{0}, \ldots, \alpha_{l}\right)$ para as quais (2.6) possui alguns tipos de soluções periódicas e exibimos essas soluções.

\subsection{Soluções Periódicas}

Recentemente, Carvalho e Ladeira [6] fizeram um estudo sobre soluções periódicas para equações da forma

$$
\dot{x}(t)=f(\alpha, x(t), x(t-1)), \quad \alpha \in \mathbb{R},
$$

onde eles mostraram que a equação (2.7) não tem soluções 1-periódicas para qualquer $f$ contínua. E se $f$ é localmente Lipschitz, eles fizeram uma nova prova da não existência de soluções 2-periódicas para a equação (2.7). Em particular, a equação

$$
\dot{x}(t)=a x(t)+b x(t-1)
$$

não tem soluções 1 ou 2 periódicas não constantes.

O teorema a seguir estende o resultado relativo a (2.8) para a equação (2.6).

Teorema 2.2.1. A equação (2.6), para $\varepsilon>0$, não possui soluções 1-periódicas ou 2-periódicas não constantes.

Prova. Pelo Lema 1.2.6, se (2.6) tivesse uma solução 2-periódica, ela seria da forma

$$
x(t)=a_{0}(t)+a_{1}(t) \cos (\pi t),
$$

com $a_{0}(t)$ e $a_{1}(t)$ 1-periódicas. Observe que uma solução 1-periódica seria da forma (2.9) com $a_{1}(t) \equiv 0$. 
Como

$$
x(t-r)=a_{0}(t)+(-1)^{r} a_{1}(t) \cos (\pi t)
$$

e

$$
\dot{x}(t)=\dot{a}_{0}(t)+\dot{a}_{1}(t) \cos (\pi t)-a_{1}(t) \pi \operatorname{sen}(\pi t)
$$

substituindo (2.9), (2.10) e (2.11) em (2.6), e usando o fato que as funções 1 , $\cos (\pi t), \operatorname{sen}(\pi t)$ são l.i. com relação às funções 1-periódicas, temos que:

a) A equação (2.6) não tem solução 1-periódica não constante. De fato, fazendo $a_{1}(t) \equiv 0, x(t)$ é solução 1-periódica da (2.6) se, e somente se, existir $a_{0}(t)$ 1-periódica tal que

$$
\varepsilon \dot{a}_{0}(t)=\left(\sum_{r=0}^{l} \alpha_{r}\right) a_{0}(t) .
$$

Como a solução geral de (2.12) é

$$
a_{0}(t)=c e^{\frac{1}{c}\left(\sum_{r=0}^{t} \alpha_{r}\right) t}, \quad c \in \mathbb{R}
$$

as únicas soluções limitadas possíveis são constantes. A afirmação (a) fica provada.

b) A equação (2.6) não possui solução 2-periódica não constante. De fato, $x(t)$ será solução 2-periódica da (2.6) se, e somente se, existirem $a_{0}(t)$ e $a_{1}(t)$ 1-periódicas tais que o sistema

$$
\begin{gathered}
\varepsilon \dot{a}_{0}(t)=\left(\sum_{r=0}^{l} \alpha_{r}\right) a_{0}(t), \\
\varepsilon\left(\dot{a}_{1}(t) \cos (\pi t)-a_{1}(t) \pi \operatorname{sen}(\pi t)\right)=\left(\sum_{r=0}^{l}(-1)^{r} \alpha_{r}\right) a_{1}(t) \cos (\pi t),
\end{gathered}
$$


esteja satisfeito. Note que o sistema (2.13) é desacoplado. Já vimos que, (2.12) implica $a_{0}(t)$ constante. Definindo $y(t)=a_{1}(t) \cos (\pi t)$ e substituindo na segunda equação de (2.13), obtemos

$$
\varepsilon \dot{y}(t)=\left(\sum_{r=0}^{l}(-1)^{r} \alpha_{r}\right) y(t) .
$$

Observe que as únicas soluções limitadas possíveis de (2.14) são constantes e, portanto, $a_{1}(t) \equiv 0$. Assim, fica provada a afirmação (b).

O teorema a seguir fornece as condições necessárias e suficientes para que (2.6) tenha soluções $p$-periódicas, $p>2$ inteiro.

Dado $p>2$ inteiro, sejam $\sigma \in \mathcal{J}_{p}:=\{1,2, \ldots,[(p-1) / 2]\}, \varepsilon>0$ real e consideramos o seguinte conjunto

$$
\begin{gathered}
\mathcal{P}_{p \sigma}^{\varepsilon}:=\left\{\left(\alpha_{0}, \ldots, \alpha_{l}\right) \in \mathbb{R}^{l+1}: \sum_{r=0}^{l} \alpha_{r} \cos \left(r w_{\sigma}\right)=0 \text { e } \sum_{r=0}^{l} \alpha_{r} \operatorname{sen}\left(r w_{\sigma}\right)=\varepsilon \rho_{\sigma k}\right. \\
\left.\operatorname{com} w_{\sigma}:=2 \sigma \pi / p, \rho_{\sigma k}:=2 k \pi-w_{\sigma} \text { e } k \in \mathbb{Z}\right\} .
\end{gathered}
$$

Teorema 2.2.2. Sejam $p>2$ inteiro, $\left(\alpha_{0}, \ldots, \alpha_{l}\right)$ tal que $\sum_{r=0}^{l} \alpha_{r} \neq 0$ e $\varepsilon>0$. A função

$$
\hat{x}_{p}^{\sigma}(t)=\hat{a}_{\sigma}(t) \cos \left(w_{\sigma} t\right)+\hat{b}_{\sigma}(t) \operatorname{sen}\left(w_{\sigma} t\right) \not \equiv 0, \quad \sigma \in \mathcal{J}_{p}
$$

onde $\hat{a}_{\sigma}(t)=c_{1 \sigma} \cos (2 k \pi t)-c_{2 \sigma} \operatorname{sen}(2 k \pi t), \hat{b}_{\sigma}(t)=c_{1 \sigma} \operatorname{sen}(2 k \pi t)+c_{2 \sigma} \cos (2 k \pi t)$, $c_{1 \sigma}, c_{2 \sigma} \in \mathbb{R}$ e $k \in \mathbb{Z}$, é solução p-periódica da equação (2.6) se, e somente se, $\left(\alpha_{0}, \ldots, \alpha_{l}\right) \in \mathcal{P}_{p \sigma}^{\varepsilon}$.

Prova. Seja $x(t)$ uma função $p$-periódica, $p>2$, de classe $C^{1}$. O Lema 1.2.6 garante que esta função pode ser escrita na forma (1.9), isto é,

$$
x(t)=a_{0}(t)+\sum_{j=1}^{\left\lfloor\frac{p}{2}\right]}\left(a_{j}(t) \cos \left(w_{j} t\right)+b_{j}(t) \operatorname{sen}\left(w_{j} t\right)\right),
$$


onde $a_{j}$ 's e $b_{j}$ 's são funções 1-periódicas, com $b_{\frac{p}{2}}(t) \equiv 0$ se $p$ for par.

Daí,

$$
\begin{gathered}
x(t-r)=a_{0}(t)+\sum_{j=1}^{\left\lfloor\frac{p}{2}\right\rfloor}\left[\left[a_{j}(t) \cos \left(r w_{j}\right)-b_{j}(t) \operatorname{sen}\left(r w_{j}\right)\right] \cos \left(w_{j} t\right)+\right. \\
\left.+\left[a_{j}(t) \operatorname{sen}\left(r w_{j}\right)+b_{j}(t) \cos \left(r w_{j}\right)\right] \operatorname{sen}\left(w_{j} t\right)\right]
\end{gathered}
$$

e

$$
\dot{x}(t)=\dot{a}_{0}(t)+\sum_{j=1}^{\left\lfloor\frac{p}{2}\right\rfloor}\left[\left[\dot{a}_{j}(t)+w_{j} b_{j}(t)\right] \cos \left(w_{j} t\right)+\left[\dot{b}_{j}(t)-w_{j} a_{j}(t)\right] \operatorname{sen}\left(w_{j} t\right)\right] .
$$

Usando a independência linear de

$$
\left\{1, \cos \left(w_{j} t\right), \operatorname{sen}\left(w_{j} t\right) ; j=1, \ldots,[p / 2]\right\},
$$

com relação às funções 1-periódicas, temos que $x(t)$ é solução $p$-periódica da (2.6) se, e somente se:

(a) quando $p$ for par, existirem funções 1-periódicas

$$
a_{0}(t), a_{\frac{p}{2}}(t), a_{j}(t), b_{j}(t), j=1, \ldots,[(p-1) / 2]
$$

satisfazendo

$$
\begin{aligned}
\varepsilon \dot{a}_{0}(t) & =\left(\sum_{r=0}^{l} \alpha_{r}\right) a_{0}(t), \\
\varepsilon\left(\dot{a}_{\frac{p}{2}}(t) \cos (\pi t)-a_{\frac{p}{2}}(t) \pi \operatorname{sen}(\pi t)\right) & =\left(\sum_{r=0}^{l}(-1)^{r} \alpha_{r}\right) a_{\frac{p}{2}}(t) \cos (\pi t)
\end{aligned}
$$

e os $[(p-1) / 2]$ sistemas $2 \times 2$ :

$$
\varepsilon\left(\begin{array}{l}
\dot{a}_{j}(t) \\
\dot{b}_{j}(t)
\end{array}\right)=A_{j}\left(\begin{array}{l}
a_{j}(t) \\
b_{j}(t)
\end{array}\right), \quad A_{j}:=\left[\begin{array}{cc}
\mu_{j} & -\nu_{\varepsilon j} \\
\nu_{\varepsilon j} & \mu_{j}
\end{array}\right], j=1, \ldots,[(p-1) / 2],
$$


onde

$$
\mu_{j}=\sum_{r=0}^{l} \alpha_{r} \cos \left(r w_{j}\right)_{l} \quad \text { e } \quad \nu_{\varepsilon j}=\varepsilon w_{j}+\sum_{r=0}^{l} \alpha_{r} \operatorname{sen}\left(r w_{j}\right) .
$$

(b) quando $p$ for ímpar, existirem funções 1-periódicas

$$
a_{0}(t), a_{j}(t), b_{j}(t), j=1, \ldots,[(p-1) / 2]
$$

satisfazendo

$$
\varepsilon \dot{a}_{0}(t)=\left(\sum_{r=0}^{l} \alpha_{r}\right) a_{0}(t)
$$

e os $[(p-2) / 2]$ sistemas $2 \times 2$ :

$$
\varepsilon\left(\begin{array}{l}
\dot{a}_{j}(t) \\
\dot{b}_{j}(t)
\end{array}\right)=A_{j}\left(\begin{array}{l}
a_{j}(t) \\
b_{j}(t)
\end{array}\right), A_{j}:=\left[\begin{array}{cc}
\mu_{j} & -\nu_{\varepsilon j} \\
\nu_{\varepsilon j} & \mu_{j}
\end{array}\right], j=1, \ldots,\left[\frac{p-1}{2}\right],
$$

onde

$$
\mu_{j}=\sum_{r=0}^{l} \alpha_{r} \cos \left(r w_{j}\right)_{\vartheta} \quad \text { e } \quad \nu_{\varepsilon j}=\varepsilon w_{j}+\sum_{r=0}^{l} \alpha_{r} \operatorname{sen}\left(r w_{j}\right) .
$$

Agora, observe que o sistema (2.18) é desacoplado e independente dos $N$ sistemas (2.19) e estes, por sua vez, também são independentes. Como, $a_{0}(t) \equiv a_{\frac{p}{2}}(t) \equiv 0$ são as únicas soluções periódicas de (2.18) ou (2.20) a prova depende apenas da análise dos sistemas (2.19), se $p$ for par, ou (2.21), se $p$ for ímpar.

Para cada $j \in \mathcal{J}_{p}$, a solução geral do $j$-ésimo sistema $2 \times 2$ em (2.19) ou (2.21) é da forma:

$$
\left(\begin{array}{l}
a_{j}(t) \\
b_{j}(t)
\end{array}\right)=e^{\frac{1}{6} A_{j} t}\left(\begin{array}{l}
c_{1 j} \\
c_{2 j}
\end{array}\right), \quad\left(\begin{array}{c}
c_{1 j} \\
c_{2 j}
\end{array}\right) \in \mathbb{R}^{2}
$$

isto é,

$$
\left(\begin{array}{l}
a_{j}(t) \\
b_{j}(t)
\end{array}\right)=e^{\frac{\mu_{j}}{\varepsilon} t}\left[\begin{array}{cc}
\cos \left(\frac{\nu_{\varepsilon j}}{\varepsilon} t\right) & -\operatorname{sen}\left(\frac{\nu_{\varepsilon j}}{\varepsilon} t\right) \\
\operatorname{sen}\left(\frac{\nu_{\varepsilon j}}{\varepsilon} t\right) & \cos \left(\frac{\nu_{\varepsilon j}}{\varepsilon} t\right)
\end{array}\right]\left(\begin{array}{l}
c_{1 j} \\
c_{2 j}
\end{array}\right)
$$


O j-ésimo sistema em (2.19) ou em (2.21) tem solução 1-periódica se, e somente se,

$$
\mu_{j}=0 \quad \text { e } \quad \nu_{\varepsilon j}=2 k \varepsilon \pi, \quad k \in \mathbb{Z}
$$

isto é, $\left(\alpha_{0}, \ldots, \alpha_{l}\right) \in \mathcal{P}_{p j}^{e}$.

Nestas condições,

$$
\begin{aligned}
& a_{j}(t)=c_{1 j} \cos (2 k \pi t)-c_{2 j} \operatorname{sen}(2 k \pi t), \\
& b_{j}(t)=c_{1 j} \operatorname{sen}(2 k \pi t)+c_{2 j} \cos (2 k \pi t) .
\end{aligned}
$$

Agora, dada a independência linear de

$$
\left\{1, \cos \left(w_{j} t\right), \operatorname{sen}\left(w_{j} t\right), j=1, \ldots,[p / 2]\right\}
$$

com relação às funções 1-periódicas, e notando que

$$
\hat{x}_{p}^{\sigma}(t)=\hat{a}_{\sigma}(t) \cos \left(w_{\sigma} t\right)+\hat{b}_{\sigma}(t) \operatorname{sen}\left(w_{\sigma} t\right), \quad \sigma \in \mathcal{J}_{p}
$$

é a única forma de expressar $\hat{x}_{p}^{\sigma}(t)$ como combinação linear de (2.24). Isto é, na expressão de $\hat{x}_{p}^{\sigma}(t), a_{j}(t), b_{j}(t) \equiv 0$ para todo $j \neq \sigma$ e $a_{\sigma}(t):=\hat{a}_{\sigma}(t), b_{\sigma}(t):=\hat{b}_{\sigma}(t)$. Portanto, $\hat{x}_{p}^{\sigma}(t)$ é solução p-periódica de $(2.6)$, se e somente se, $\left(\alpha_{0}, \ldots, \alpha_{l}\right) \in$ $\mathcal{P}_{p \sigma}^{\varepsilon}$.

Corolário 2.2.1. Sejam $p>2, k \in \mathbb{Z}, \sigma \in \mathcal{J}_{p},\left(\alpha_{0}, \ldots, \alpha_{l}\right) \in \mathcal{P}_{p \sigma}^{\varepsilon}$ e $q=$ $|k p-\sigma|$. Se $p$ e q são relativamente primos, então a solução p-periódica de (2.6) no Teorema 2.2.2 também é $\frac{p}{q}$-periódica.

Prova. Basta observar que podemos escrever $\hat{x}_{p}^{\sigma}(t)$ na forma

$$
c_{1 \sigma} \cos \left(2 \pi \frac{k p-\sigma}{p} t\right)-c_{2 \sigma} \operatorname{sen}\left(2 \pi \frac{k p-\sigma}{p} t\right)
$$

ou seja, $\hat{x}_{p}^{\sigma}(t)$ é uma combinação linear (usual) de $\cos \left(2 \frac{q}{p} \pi t\right)$ e sen $\left(2 \frac{q}{p} \pi t\right)$. 


\subsection{Hipersuperfícies de Bifurcação}

Observe que, fixados $m, n, 0 \leq m<n \leq l$, o conjunto $\mathcal{P}_{p j}^{\varepsilon}$ dado no Teorema 2.2.2 pode ser visto como

$$
\left(\bigcup_{\substack{s=1 \\ k \in \mathbb{Z}}}^{n-m} \Gamma_{s k}^{\varepsilon}\right) \cap \mathcal{H}_{p}^{j}
$$

onde $\mathcal{H}_{p}^{j}$ é o hiperplano dado por

$$
\left\{\left(\alpha_{0}, \ldots, \alpha_{l}\right) \in \mathbb{R}^{l+1}: \sum_{r=0}^{l} \alpha_{r} \cos \left(r w_{j}\right)=0\right\},
$$

e $\Gamma_{s k}^{\varepsilon}$ são as hipersuperfícies definidas da seguinte forma: $\Gamma_{s k}^{\varepsilon}:=\Gamma_{s k}^{\varepsilon}(t, \omega) \in \mathbb{R}^{l+1}$, $t:=\left(t_{0}, \ldots, \hat{t}_{m}, \ldots, \hat{t}_{n}, \ldots, t_{l}\right) \in \mathbb{R}^{l-1}$, (onde ^ significa que a respectiva coordenada ou índice acentuado deve ser excluído), $\omega \in\left(\frac{(n-m) k+s-1}{n-m} \pi, \frac{(n-m) k+s}{n-m} \pi\right)$, $s=1, \ldots, n-m$ e $\Gamma_{s k}^{\varepsilon}=\left(\alpha_{0}, \ldots, \alpha_{l}\right)$ é definida por

$$
\Gamma_{s k}^{\varepsilon}:\left\{\begin{array}{c}
\alpha_{r}=t_{r}, t_{r} \in \mathbb{R}, r=0,1, \ldots, \hat{m}, \ldots, \hat{n}, \ldots, l \\
\alpha_{m}=-\frac{\varepsilon(2 k \pi-\omega) \cos (n \omega)+\sum_{\substack{r=0 \\
r \neq m, n}}^{l} t_{r} \operatorname{sen}((n-r) w)}{\operatorname{sen}((n-m) \omega)} \\
\alpha_{n}=\frac{\varepsilon(2 k \pi-\omega) \cos (m \omega)+\sum_{\substack{r=0 \\
r \neq m, n}}^{l} t_{r} \operatorname{sen}((m-r) w)}{\operatorname{sen}((n-m) \omega)}
\end{array}\right.
$$

Definição 2.3.1. A k-ésima hipersuperfície, dada por $\cup_{s=1}^{n-m} \Gamma_{s k}^{\varepsilon}, k \in \mathbb{Z}, \varepsilon>0$, parametrizada pelas equações (2.25), é chamada de $k$-hipersuperfície de bifurcação de (2.6).

A definição é sugerida pelo fato da $k$-ésima hipersuperfície, no espaço dos coeficientes de (2.6), ser o lugar geométrico dos $\left(\alpha_{0}, \ldots, \alpha_{l}\right)$ tais que existe uma raiz característica imaginária pura. Isto significa que quando cruzamos uma tal hipersuperfície, há perda ou ganho de estabilidade. 
De fato, a equação característica de (2.6) é

$$
\varepsilon \lambda=\sum_{r=0}^{l} \alpha_{r} e^{-\lambda r}
$$

fazendo $\lambda=\theta+\omega i, \theta, \omega \in \mathbb{R}$, obtemos

$$
\varepsilon \theta=\sum_{r=0}^{l} \alpha_{r} e^{-\theta r} \cos (r \omega) \quad \text { e } \quad \varepsilon \omega=-\sum_{r=0}^{l} \alpha_{r} e^{-\theta r} \operatorname{sen}(r \omega) .
$$

Agora, tomando $\theta=0$ temos

$$
\sum_{r=0}^{l} \alpha_{r} \cos (r \omega)=0, \quad \text { e } \quad \varepsilon \omega=-\sum_{r=0}^{l} \alpha_{r} \operatorname{sen}(r \omega) .
$$

Se $m, n$ são inteiros tais que $0 \leq m<n \leq l$, temos da segunda equação que

$$
\alpha_{m}=-\frac{\varepsilon \omega+\sum_{\substack{r=0 \\ r \neq m, n}}^{l} \alpha_{r} \operatorname{sen}(r \omega)+\alpha_{n} \operatorname{sen}(n \omega)}{\operatorname{sen}(m \omega)} .
$$

Substituindo $\alpha_{m}$ na primeira equação, obtemos

$$
\alpha_{n}=\frac{-\varepsilon \omega \cos (m \omega)+\sum_{\substack{r=0 \\ r \neq m, n}}^{l} \alpha_{r} \operatorname{sen}((m-r) \omega)}{\operatorname{sen}((n-m) \omega)} .
$$

Voltando à expressão de $\alpha_{m}$, temos

$$
\alpha_{m}=-\frac{-\varepsilon \omega \cos (n \omega)+\sum_{\substack{r=0 \\ r \neq m, n}}^{l} \alpha_{r} \operatorname{sen}((n-r) \omega)}{\operatorname{sen}((n-m) \omega)} .
$$

Assim,

$$
\left\{\begin{aligned}
\alpha_{m} & =-\frac{-\varepsilon \omega \cos (n \omega)+\sum_{\substack{r \neq m \\
r \neq m, n}}^{l} \alpha_{r} \operatorname{sen}((n-r) \omega)}{\operatorname{sen}((n-m) \omega)}, \\
\alpha_{n} & =\frac{-\varepsilon \omega \cos (m \omega)+\sum_{\substack{r=0 \\
r \neq m, n}}^{l} \alpha_{r} \operatorname{sen}((m-r) \omega)}{\operatorname{sen}((n-m) \omega)}, \quad w \neq \frac{k \pi}{n-m}, k \in \mathbb{Z} .
\end{aligned}\right.
$$

Fazendo a mudança de variável $\omega \leftrightarrow 2 k \pi-\omega$, obtemos (2.25). 


\section{Exemplos}

Exemplo 2.3.1. Fixando $l=1$ e $\varepsilon=1$ em (2.6), obtemos

$$
\dot{x}(t)=\alpha_{0} x(t)+\alpha_{1} x(t-1) .
$$

Neste caso, as $k$-ésimas hipersuperfícies (curvas) de bifurcação são dadas por

$$
\left\{\begin{array}{l}
\alpha_{0}=-(2 k \pi-\omega) \frac{\cos \omega}{\operatorname{sen} \omega}, \\
\alpha_{1}=(2 k \pi-\omega) \frac{1}{\operatorname{sen} \omega}, \quad \omega \in(0, \pi) .
\end{array}\right.
$$

$\mathrm{O}$ conjunto $\mathcal{P}_{p j}^{1}$, que neste caso é discreto, pode ser visto na Figura 2.1 como interseção dos hiperplanos (retas) $\mathcal{H}_{p}^{j}$ com as hipersuperfícies (curvas) $\Gamma_{k}$.

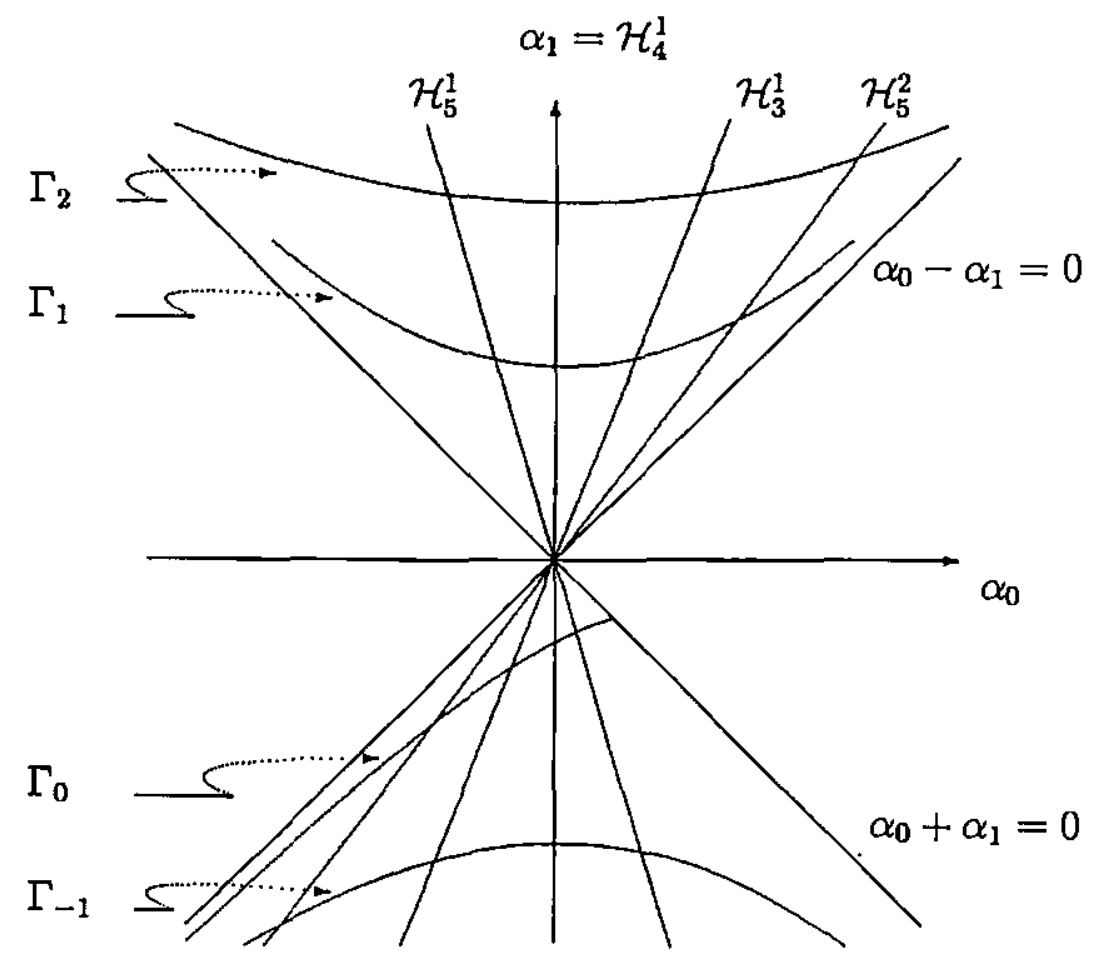

Figura 2.1: $\mathcal{P}_{p j}^{1}=\Gamma_{k} \cap \mathcal{H}_{p}^{j}, \quad k=-1,0,1,2$ para cada $p \in\{3,4,5\}$. 
O exemplo seguinte é importante porque a equação limite $(\varepsilon=0)$ tem sido bastante estudada na área das equações a diferenças, veja [4], [23], por exemplo.

Exemplo 2.3.2. Fixaremos $l=2$ e $\alpha_{0}=-1$ em (2.6), isto é,

$$
\varepsilon \dot{x}(t)+x(t)=\alpha_{1} x(t-1)+\alpha_{2} x(t-2) .
$$

Neste caso, as $k$-ésimas hipersuperfícies (curvas) de bifurcação são dadas por

$$
\left\{\begin{array}{l}
\alpha_{1}=-\varepsilon(2 k \pi-\omega) \frac{\cos (2 \omega)}{\operatorname{sen} \omega}+2 \cos \omega, \\
\alpha_{2}=\varepsilon(2 k \pi-\omega) \frac{\cos w}{\operatorname{sen} \omega}-1, \quad \omega \in(0, \pi) .
\end{array}\right.
$$

Representamos o conjunto $\mathcal{P}_{3,1}^{\varepsilon}$ na figura que se segue.

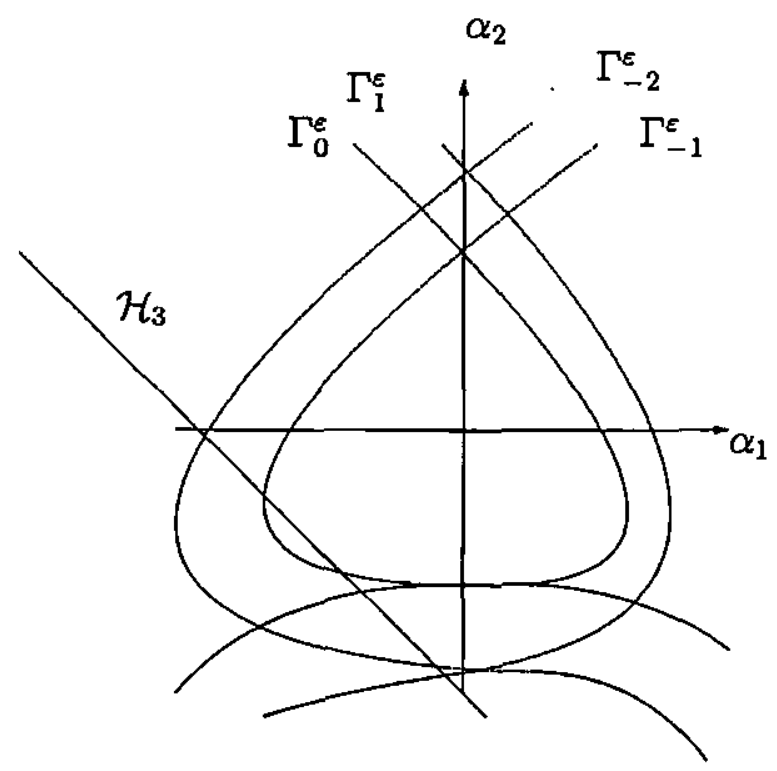

Figura 2.2: $O$ conjunto $\mathcal{P}_{3,1}^{\varepsilon}$ é a interseção das hipersuperfícies (curvas) de bifurcação $\Gamma_{k^{\prime} s}^{\epsilon} \operatorname{com}$ a reta $\mathcal{H}_{3}^{1}$.

Mais adiante, motivados pelo Exemplo 2.3.2, veremos que ao considerarmos as equações a diferenças como caso limite de um problema de perturbação singular, 
podemos enquadrar resultados conhecidos de equações a diferenças num contexto mais geral. Além disso, o Teorema 3.1.1 a seguir, nos permite generalizar alguns resultados para um número arbitrário de retardamentos.

Daqui para frente representamos somente as hipersupefícies de bifurcação.

Exemplo 2.3.3. Fixaremos $l=3$ em (2.6), isto é,

$$
\varepsilon \dot{x}(t)=\alpha_{0} x(t)+\alpha_{1} x(t-1)+\alpha_{2} x(t-2)+\alpha_{3} x(t-3) .
$$

Em cada um dos ítens 1) - 4) que se seguem fixaremos $\alpha_{r}=0, r=3,2,1$, respectivamente. Representaremos nas figuras $2.3-2.6$, em (a) $\varepsilon=1$, em (b) $\varepsilon=0.1$, em (c) $\varepsilon=0.01 \mathrm{e} \mathrm{em} \mathrm{(d)} \varepsilon=0.001$, com o objetivo de transmitir uma idéia de como as superfícies de bifurcação se deformam quando $\varepsilon \rightarrow 0$.

Assim, consideramos os seguintes casos:

1) Se $\alpha_{3}=0$ (observe que $t_{3}=0 \mathrm{em}(2.25)$ ), temos dois casos: 
- escolhendo $m=0$ e $n=1$, as superfícies são dadas por

$$
\left\{\begin{array}{l}
\alpha_{0}=-\varepsilon(2 k \pi-\omega) \frac{\cos w}{\operatorname{sen} \omega}+t_{2}, \\
\alpha_{1}=\varepsilon(2 k \pi-\omega) \frac{1}{\operatorname{sen} \omega}-2 t_{2} \cos \omega, \\
\alpha_{2}=t_{2}, \quad \omega \in(0, \pi) \quad \text { e } \quad t_{2} \in \mathbb{R} .
\end{array}\right.
$$

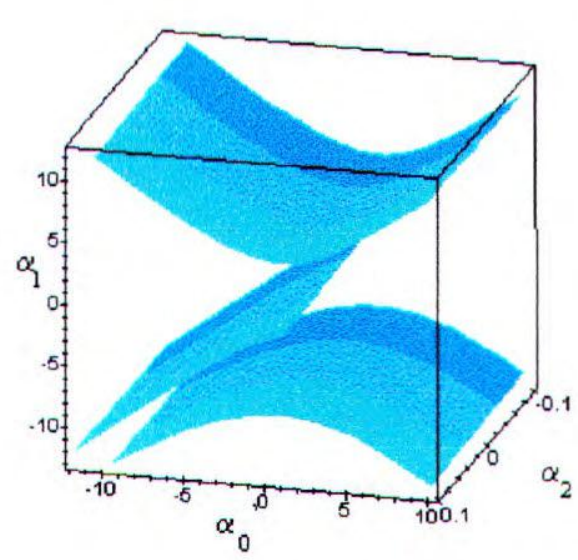

(a)

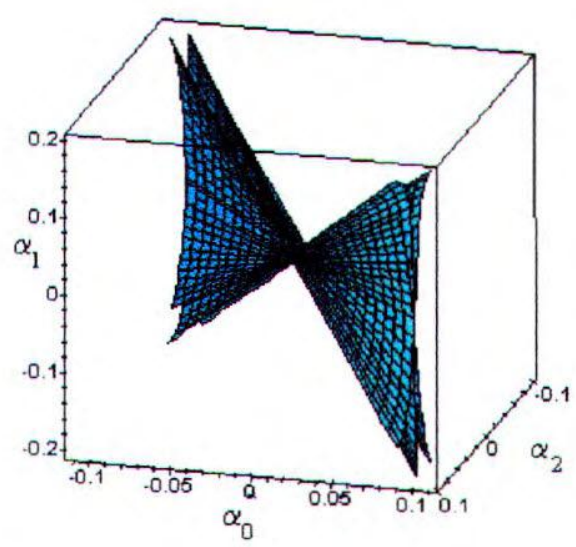

(c)

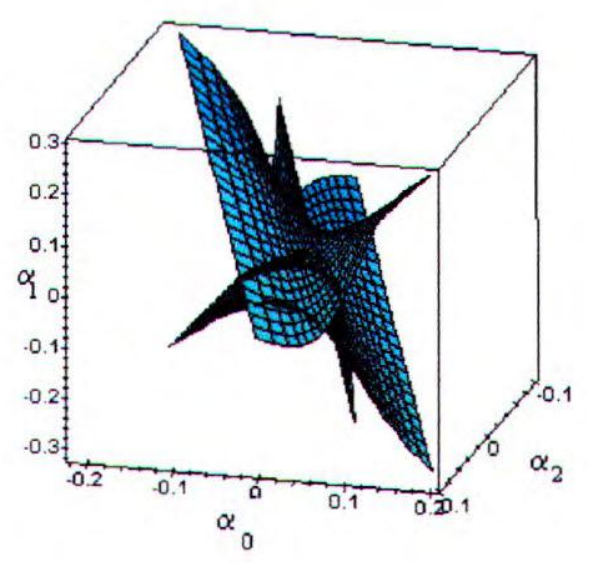

(b)

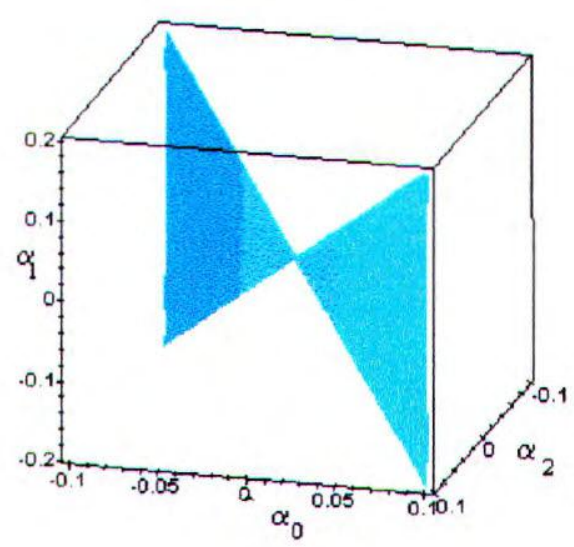

(d)

Figura 2.3.

Observe que a Fig.2.1 é o corte da Fig.2.3(a) em $\alpha_{2}=0$. Assim podemos imaginar como ficam os planos $\mathcal{H}_{p}^{j}$ na Fig.2.3. 
- escolhendo $m=1$ e $n=2$, obtemos (veja a figura 2.4)

$$
\left\{\begin{array}{l}
\alpha_{0}=t_{0}, \\
\alpha_{1}=-\varepsilon(2 k \pi-\omega) \frac{\cos (2 \omega)}{\operatorname{sen} \omega}+2 t_{0} \cos \omega, \\
\alpha_{2}=\varepsilon(2 k \pi-\omega) \frac{\cos \omega}{\operatorname{sen} \omega}+t_{0}, \quad \omega \in(0, \pi) \quad \text { e } t_{0} \in \mathbb{R},
\end{array}\right.
$$

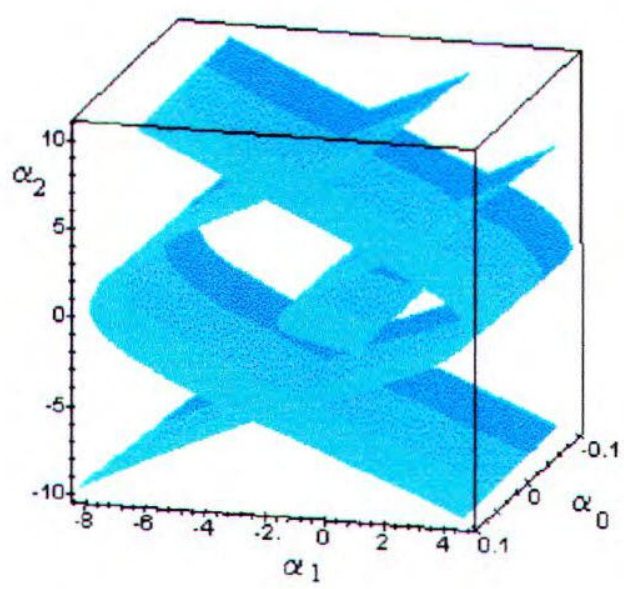

(a)

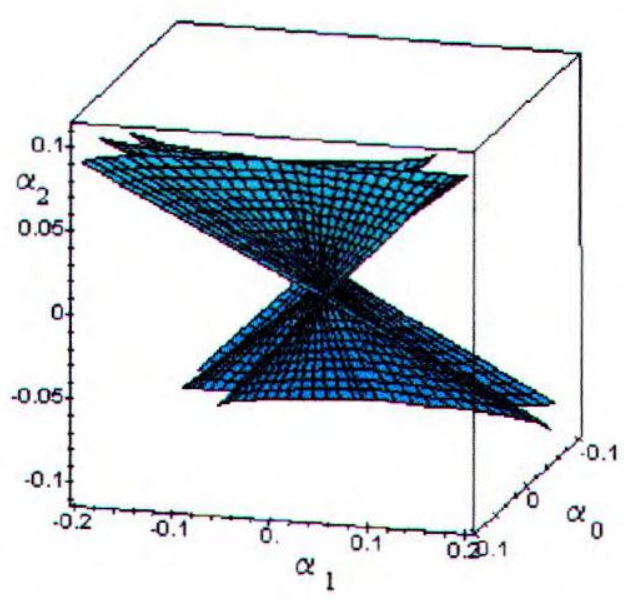

(c)

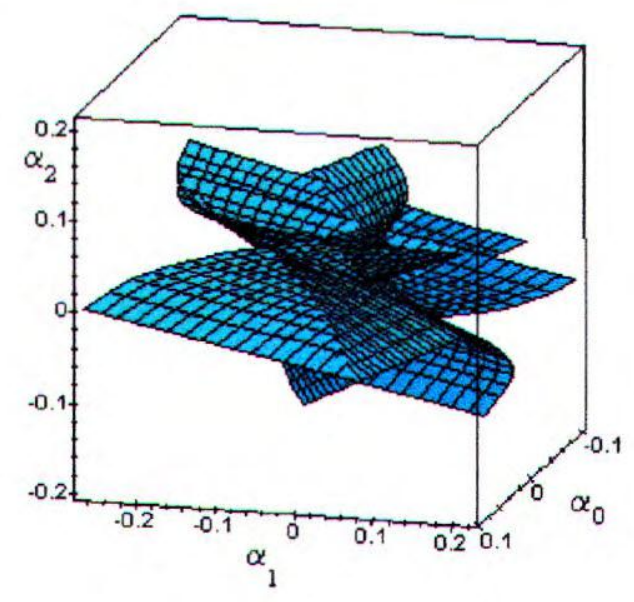

(b)

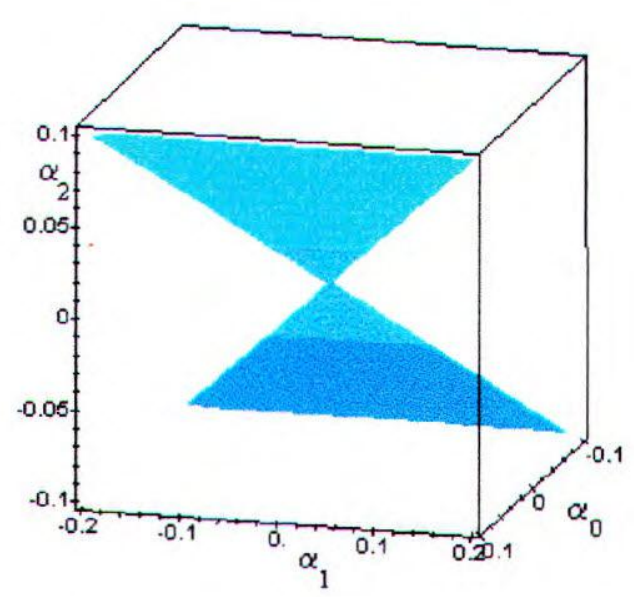

(d)

Figura 2.4.

Observe que a Fig.2.2 é o corte da Fig.2.4(a) em $\alpha_{0}=0$. 
2) Se $\alpha_{2}=0\left(t_{2}=0\right.$ em (2.25)), $m=1$ e $n=3$, temos (veja a figura 2.5.)

$$
\left\{\begin{array}{l}
\alpha_{0}=t_{0}, \\
\alpha_{1}=-\frac{\varepsilon(2 k \pi-\omega) \cos (3 \omega)+t_{0} \operatorname{sen}(3 \omega)}{\operatorname{sen}(2 \omega)}, \\
\alpha_{3}=\frac{\varepsilon(2 k \pi-\omega) \cos \omega+t_{0} \operatorname{sen} \omega}{\operatorname{sen}(2 \omega)}, \quad \omega \in\left(0, \frac{\pi}{2}\right) \cup\left(\frac{\pi}{2}, \pi\right) \text { e } t_{0} \in \mathbb{R},
\end{array}\right.
$$

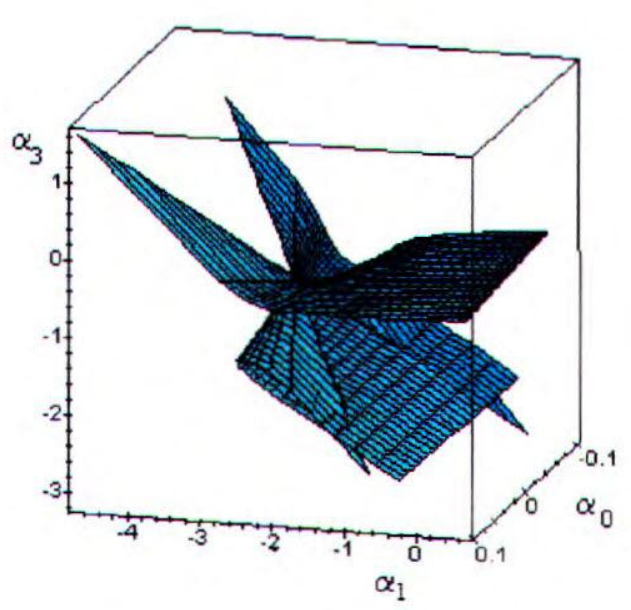

(a)

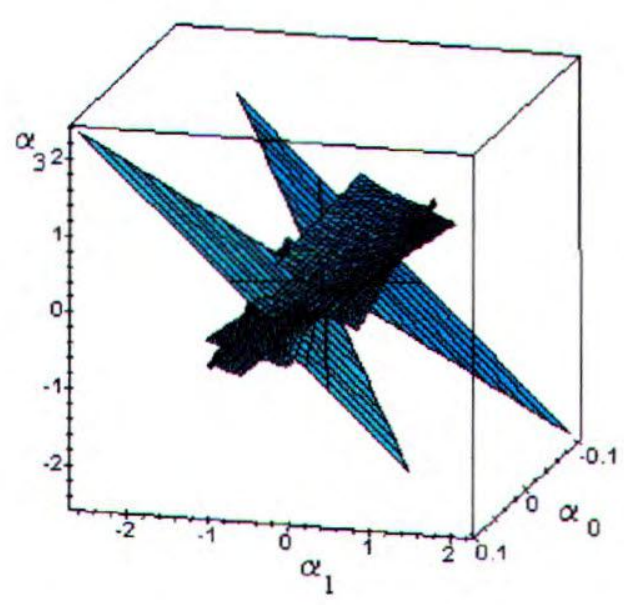

(c)

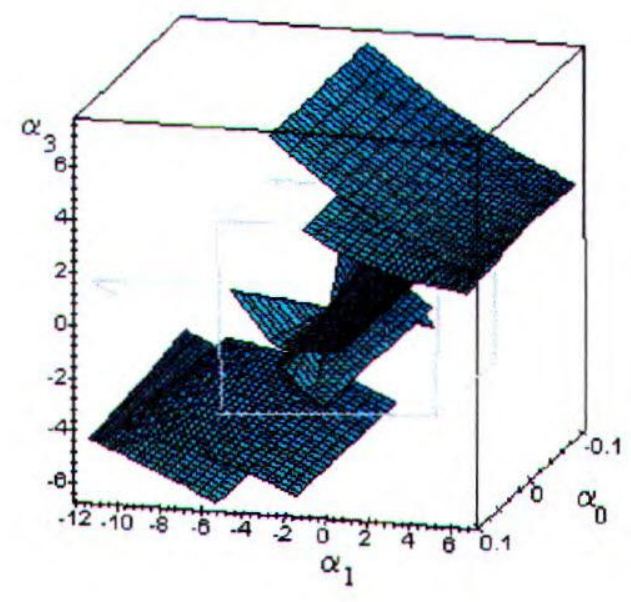

(b)

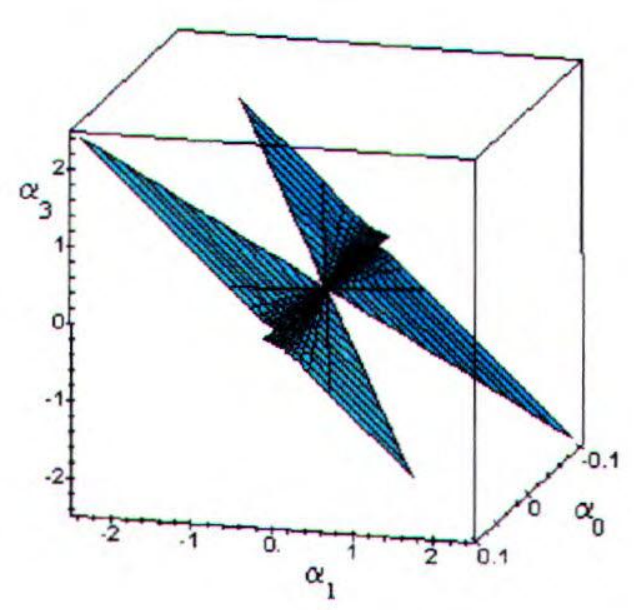

(d)

Figura 2.5. 
3) Para $\alpha_{1}=0, m=2$ e $n=3$, temos (veja a figura 2.6.)

$$
\left\{\begin{array}{l}
\alpha_{0}=t_{0}, \\
\alpha_{2}=-\frac{\varepsilon(2 k \pi-\omega) \cos (3 \omega)+t_{0} \operatorname{sen}(3 \omega)}{\operatorname{sen} \omega}, \\
\alpha_{3}=\frac{\varepsilon(2 k \pi-\omega) \cos (2 \omega)+t_{0} \operatorname{sen}(2 \omega)}{\operatorname{sen} \omega}, \quad \omega \in(0, \pi) \quad \text { e } t_{0} \in \mathbb{R},
\end{array}\right.
$$

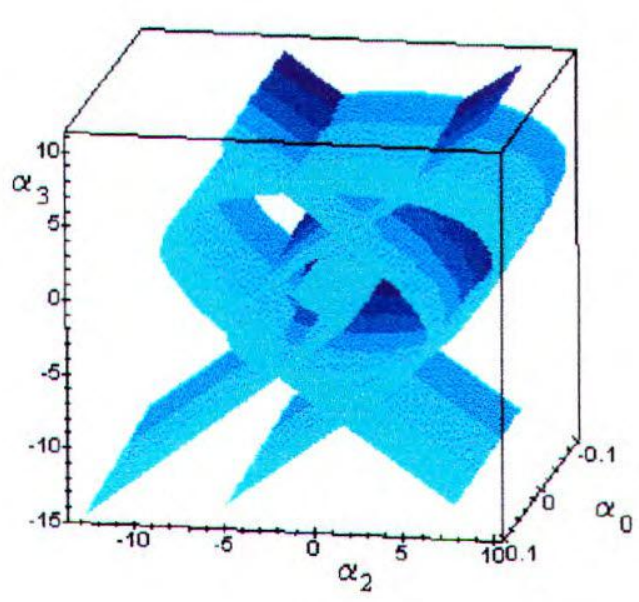

(a)

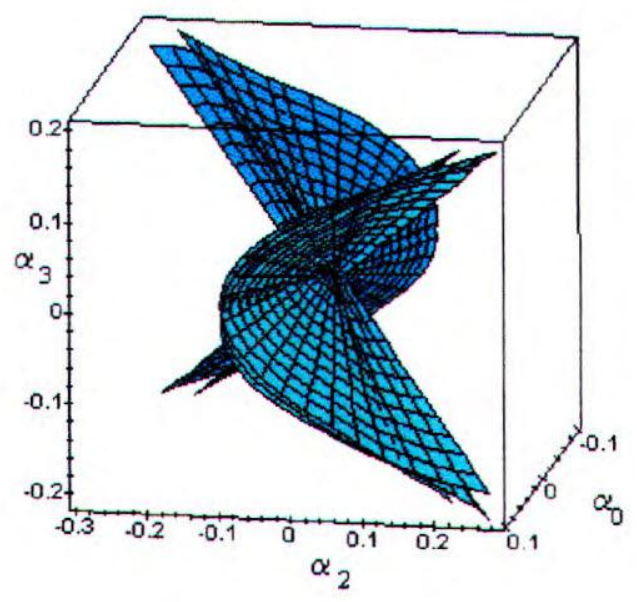

(c)

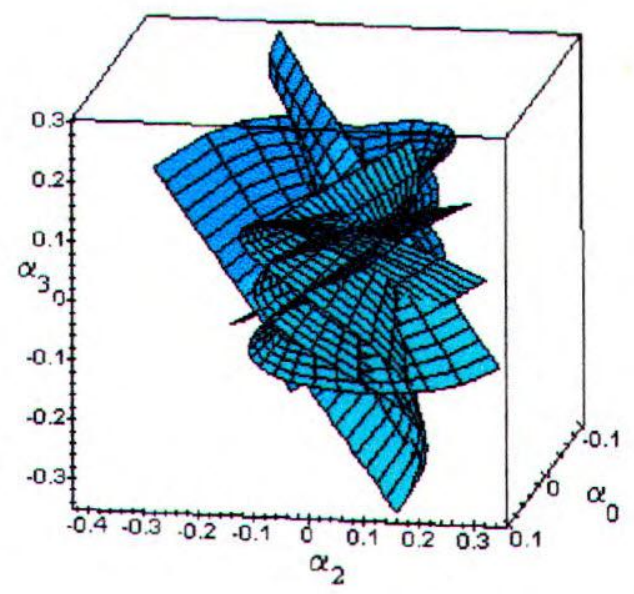

(b)

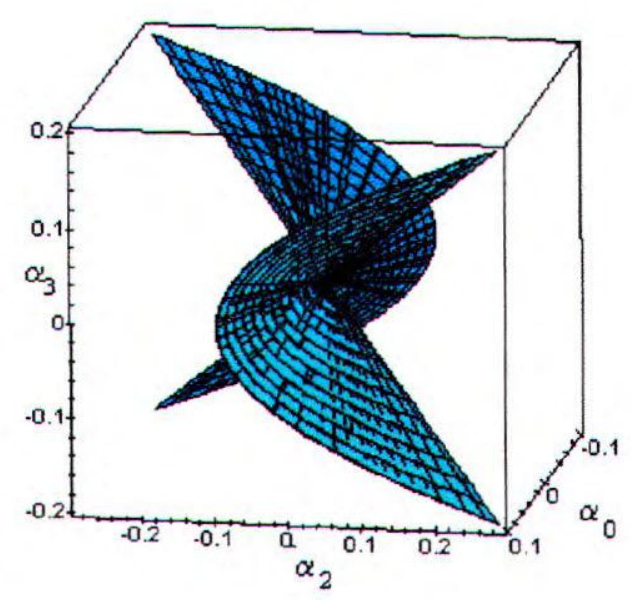

(d)

Figura 2.6. 


\section{Capítulo 3}

\section{A Equação Limite}

Neste capítulo vamos estudar a equação limite de (2.6). Isto é, o limite formal da equação (2.6), para $\varepsilon \rightarrow 0$ :

$$
\sum_{r=0}^{l} \alpha_{r} x(t-r)=0 .
$$

No capítulo subsequente estaremos particularmente interessados em estabelecer relações entre propriedades de (3.1) e de (2.6), para $\varepsilon>0$ pequeno.

\subsection{Soluções Periódicas}

O teorema abaixo nos fornece condições necessárias e suficientes para que (3.1) tenha soluções $p$-periódicas, $p \geq 1$. No caso $p>2$ ele pode ser visto como uma versão no presente contexto do Teorema 2.2.2.

Teorema 3.1.1. (a) $\hat{x}_{1}(t)=\hat{a}_{0}(t)$ é uma solução de (3.1) não constante, qualquer que seja a função 1-periódica não constante $\hat{a}_{0}(t)$ se, e somente se, $\sum_{r=0}^{l} \alpha_{r}=0$.

(b) $\hat{x}_{2}(t)=\hat{a}_{1}(t) \cos \pi t$ é uma solução 2-periódica de (3.1), qualquer que seja a função 1-periódica não trivial $\hat{a}_{1}(t)$ se, e somente se, $\sum_{r=0}^{l}(-1)^{r} \alpha_{r}=0$. 
(c) $\hat{x}_{2}^{\star}(t)=\hat{a}_{0}(t)+\hat{a}_{1}(t) \cos \pi t$ quaisquer que sejam $\hat{a}_{0}(t), \hat{a}_{1}(t)$ funções 1-periódicas, $\hat{a}_{0}(t)$ não constante ou $\hat{a}_{1}(t)$ não trivial, é uma solução 2-periódica para (3.1) se, e somente se, $\sum_{r=0}^{l} \alpha_{r}=0$ e $\sum_{r=0}^{l}(-1)^{r} \alpha_{r}=0$.

(d) Suponha que $\sum_{r=0}^{l} \alpha_{r} \neq 0$ e $\sum_{r=0}^{l}(-1)^{r} \alpha_{r} \neq 0$. Então, a função

$$
\hat{x}_{p}^{\sigma}(t)=\hat{a}_{\sigma}(t) \cos \left(w_{\sigma} t\right)+\hat{b}_{\sigma}(t) \operatorname{sen}\left(w_{\sigma} t\right), \quad \sigma \in \mathcal{J}_{p},
$$

onde $\hat{a}_{\sigma}(t), \hat{b}_{\sigma}(t)$ são funções 1-periódicas não triviais quaisquer, é solução p-periódica de (3.1) se, e somente se, $\left(\alpha_{0}, \ldots, \alpha_{l}\right) \in \mathcal{P}_{p \sigma}^{0}$.

Prova. De forma análoga ao que foi feito na prova dos Teoremas 2.2 .1 e 2.2.2, vamos considerar $x(t)$ uma função $p$-periódica, $p \geq 1$. O Lema 1.2.6 garante que $x(t)$ pode ser escrita na forma (1.9), isto é,

$$
x(t)=a_{0}(t)+\sum_{j=1}^{\left[\frac{p}{2}\right]}\left(a_{j}(t) \cos \left(w_{j} t\right)+b_{j}(t) \operatorname{sen}\left(w_{j} t\right)\right),
$$

onde $a_{j}$ 's e $b_{j}$ 's são funções 1-periódicas, com $b_{\frac{p}{2}}(t) \equiv 0$ se $p$ for par.

Daí,

$$
\begin{gathered}
x(t-r)=a_{0}(t)+\sum_{j=1}^{\left[\frac{p}{2}\right]}\left[\left[a_{j}(t) \cos \left(r w_{j}\right)-b_{j}(t) \operatorname{sen}\left(r w_{j}\right)\right] \cos \left(w_{j} t\right)+\right. \\
\left.+\left[a_{j}(t) \operatorname{sen}\left(r w_{j}\right)+b_{j}(t) \cos \left(r w_{j}\right)\right] \operatorname{sen}\left(w_{j} t\right)\right]
\end{gathered}
$$

Usando a independência linear de

$$
\left\{1, \cos \left(w_{j} t\right), \operatorname{sen}\left(w_{j} t\right), j=1, \ldots,[p / 2]\right\}
$$

com relação às funções 1-periódicas, temos que $x(t)$ é solução $p$-periódica da (3.1) se, e somente se:

(a) quando $p$ for par, existirem funções 1-periódicas

$$
a_{0}(t), a_{\frac{p}{2}}(t), a_{j}(t), b_{j}(t), j=1, \ldots,[(p-1) / 2]
$$


satisfazendo

$$
\begin{aligned}
& \left(\sum_{r=0}^{l} \alpha_{r}\right) a_{0}(t)=0, \\
& \left(\sum_{r=0}^{l}(-1)^{r} \alpha_{r}\right) a_{\frac{g}{2}}(t) \cos (\pi t)=0
\end{aligned}
$$

e os $[(p-2) / 2]$ sistemas $2 \times 2$ :

$$
\left[\begin{array}{cc}
\mu_{j} & -\nu_{0 j} \\
\nu_{0 j} & \mu_{j}
\end{array}\right]\left(\begin{array}{l}
a_{j}(t) \\
b_{j}(t)
\end{array}\right)=0, \quad j=1, \ldots,[(p-1) / 2],
$$

onde

$$
\mu_{j}=\sum_{r=0}^{l} \alpha_{r} \cos \left(r w_{j}\right), \quad \text { e } \quad \nu_{0 j}=\sum_{r=0}^{l} \alpha_{r} \operatorname{sen}\left(r w_{j}\right) .
$$

(b) quando $p$ for ímpar, existirem funções 1-periódicas

$$
a_{0}(t), a_{j}(t), b_{j}(t), j=1, \ldots,[(p-1) / 2],
$$

satisfazendo

$$
\varepsilon \dot{a}_{0}(t)=\left(\sum_{r=0}^{\ell} \alpha_{r}\right) a_{0}(t)
$$

e os $[(p-1) / 2]$ sistemas $2 \times 2$ :

$$
\left[\begin{array}{cc}
\mu_{j} & -\nu_{0 j} \\
\nu_{0 j} & \mu_{j}
\end{array}\right]\left(\begin{array}{l}
a_{j}(t) \\
b_{j}(t)
\end{array}\right), \quad j=1, \ldots,\left[\frac{p-1}{2}\right],
$$

onde

$$
\mu_{j}=\sum_{r=0}^{l} \alpha_{r} \cos \left(r w_{j}\right), \quad \text { e } \quad \nu_{0 j}=\sum_{r=0}^{l} \alpha_{r} \operatorname{sen}\left(r w_{j}\right) .
$$

Para cada $j$, o sistema (3.5) ou (3.7) admite solução não trivial se, e somente se,

$$
\operatorname{det}\left[\begin{array}{cc}
\mu_{j} & -\nu_{0 j} \\
\nu_{0 j} & \mu_{j}
\end{array}\right]=\mu_{j}^{2}+\nu_{0 j}^{2}=0
$$


isto é,

$$
\mu_{j}=\sum_{r=0}^{l} \alpha_{r} \cos \left(r w_{j}\right)=0 \quad \text { e } \quad \nu_{0 j}=\sum_{r=0}^{l} \alpha_{r} \operatorname{sen}\left(r w_{j}\right)=0,
$$

o que significa que $\left(\alpha_{0}, \ldots, \alpha_{l}\right) \in \mathcal{P}_{p j}^{0}$.

Nestas condições, qualquer par de funções 1-periódicas é solução do sistema (3.5) ou (3.7). Assim, dada a independência linear de (2.24) com relação às funções 1-periódicas, e notando que

$$
\hat{x}_{p}^{\sigma}(t)=\hat{a}_{\sigma}(t) \cos \left(w_{\sigma} t\right)+\hat{b}_{\sigma}(t) \operatorname{sen}\left(w_{\sigma} t\right), \quad \sigma \in \mathcal{J}_{p}
$$

é a única forma de expressar $\hat{x}_{p}^{\sigma}(t)$ como combinação linear de (2.24), temos que $\hat{x}_{p}^{\sigma}(t)$ é solução $p$-periódica de (3.1) se, e somente se, $\left(\alpha_{0}, \ldots, \alpha_{l}\right) \in \mathcal{P}_{p \sigma}^{0}$. Provando assim, o item (d) do teorema.

Provemos agora o ítem (a), a primeira equação em (3.4) admite solução $a_{0}(t)$ não trivial se, e somente se,

$$
\sum_{r=0}^{l} \alpha_{r}=0
$$

Neste caso, toda função $a_{0}(t)$ é solução da primeira equação em (3.4). Assim, a única forma de expressar $\hat{x}_{1}(t)$ é

$$
\hat{x}_{1}(t)=\hat{a}_{0}(t)
$$

com $\hat{a}_{0}(t):=a_{0}(t) \not \equiv 0$, 1-periódica. Isto corresponde a tomar $a_{j}(t), b_{j}(t) \equiv 0$, $j=1, \ldots,[p / 2]$, em (3.2). Ficando assim, provado o ítem (a) do teorema.

Vamos agora provar o ítem (b). Observe que a segunda equação em (3.4) pode ser escrita na forma

$$
\left(\sum_{r=0}^{l}(-1)^{r} \alpha_{r}\right) y(t)=0
$$


onde $y(t)=a_{\frac{p}{2}}(t) \cos (\pi t)$. Assim, como no caso anterior, quaquer função $y(t)$ é solução de (3.8) se, e somente se,

$$
\sum_{r=0}^{l}(-1)^{r} \alpha_{r}=0 .
$$

$\mathrm{E}$ a única forma de expressar $\hat{x}_{2}(t)$ é

$$
\hat{x}_{1}(t)=\hat{a}_{1}(t) \cos (\pi t)
$$

com $\hat{a}_{1}(t):=a_{1}(t) \not \equiv 0$, 1-periódica. Isto corresponde a tomar $a_{0}(t) \equiv 0$, $a_{j}(t), b_{j}(t) \equiv 0, j=1, \ldots,[p / 2]$, em (3.2). Provamos assim, o ítem (b) do teorema.

O ítem (c) segue dos ítens (a) e (b).

Exemplo 3.1.1. Fixaremos $l=2$ e $\alpha_{0}=-1$ em (3.1), isto é,

$$
x(t)=\alpha_{1} x(t-1)+\alpha_{2} x(t-2) .
$$

Neste caso, a curva de bifurcação é o segmento dado por

$$
\Gamma:=\Gamma_{1}^{0}:\left\{\begin{array}{l}
\alpha_{1}=2 \cos \omega, \\
\alpha_{2}=-1, \quad \omega \in(0, \pi),
\end{array}\right.
$$

e as retas correspondentes aos hiperplanos são

$$
\mathcal{H}_{p}^{j}: \alpha_{1} \cos w_{j}+\alpha_{2} \cos \left(2 w_{j}\right)=1, \quad j \in \mathcal{J}_{p}
$$

Representaremos, na figura que se segue, o conjunto $\mathcal{P}_{3,1}^{0}$ e as retas $r_{1}: \alpha_{1}+$ $\alpha_{2}=1$ e $r_{2}:-\alpha_{1}+\alpha_{2}=1$ que são os lugares onde a equação tem soluções 1-periódicas e 2-periódicas, respectivamente. Compare com o Exemplo 2.3.2. 


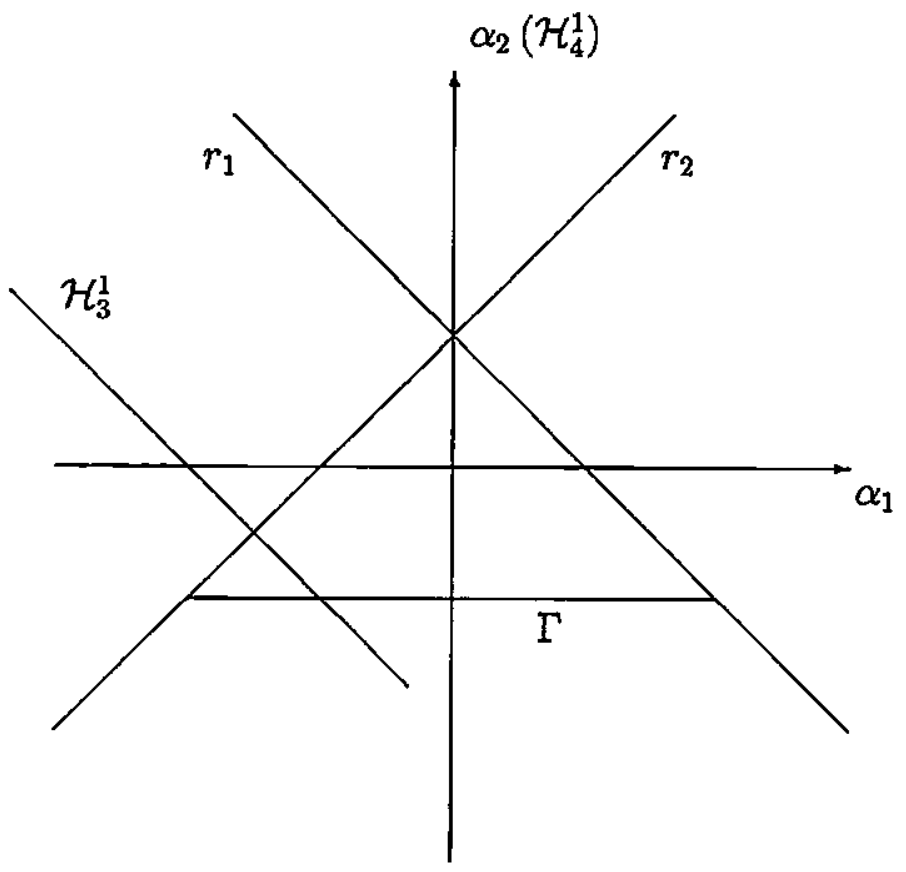

Figura 3.1: $\mathrm{O}$ conjunto $\mathcal{P}_{3,1}^{0}$ é a interseção da curva $\Gamma \operatorname{com} \mathcal{H}_{3}^{1}: \alpha_{1}+\alpha_{2}=2$. $\mathrm{O}$ conjunto $\mathcal{P}_{4,1}^{0}$ é a interseção de $\Gamma \operatorname{com} \mathcal{H}_{4}^{1}: \alpha_{1}=0$. As retas $r_{1}$ e $r_{2}$ são os lugares onde (2.27) tem soluções 1 e 2-periódicas, respectivamente.

\subsection{Um Caso em que Soluçōes são Periódicas}

Se $\alpha_{0} \neq 0$, podemos re-escrever (3.1) na forma

$$
x(t)=\sum_{r=1}^{l} \beta_{r} x(t-r), \text { onde } \beta_{r}=-\alpha_{r} / \alpha_{0}
$$

e (3.1) passa a ser caracterizada pelos $l$ coeficientes $\beta_{1}, \ldots, \beta_{l}$. Assim, dado $p>2$ inteiro, o conjunto $\mathcal{P}_{p j}^{0}$ é agora entendido como um subconjunto do $\mathbb{R}^{l}$ dado por:

$$
\begin{aligned}
& \left\{\left(\beta_{1}, \ldots, \beta_{l}\right) \in \mathbb{R}^{l}: \sum_{r=1}^{l} \beta_{r} \cos \left(r w_{j}\right)=1 \text { e } \sum_{r=1}^{l} \beta_{r} \operatorname{sen}\left(r w_{j}\right)=0\right. \\
& \left.\operatorname{com} j \in \mathcal{J}_{p}, \text { e } w_{j}:=2 j \pi / p\right\}
\end{aligned}
$$


e fixando $m, n, 0 \leq m<n \leq l$, o conjunto $\mathcal{P}_{p j}^{0}$ pode ser visto como

$$
\left(\bigcup_{s=1}^{n-m} \Gamma_{s}^{0}\right) \cap \mathcal{H}_{p}^{j}
$$

onde $\Gamma_{s}^{0}$ são as hipersuperfícies parametrizadas por

$$
\Gamma_{s}^{0}:=\Gamma_{s}^{0}(t, \omega) \in \mathbb{R}^{l},
$$

$t:=\left(t_{1}, \ldots, \hat{t}_{m}, \ldots, \hat{t}_{n}, \ldots, t_{l}\right) \in \mathbb{R}^{l-2}, \omega \in\left(\frac{s-1}{n-m} \pi, \frac{s}{n-m} \pi\right), s=1, \ldots, n-m \mathrm{e}$ $\Gamma_{s}^{0}=\left(\beta_{1}, \ldots, \beta_{l}\right)$ é definida por

$$
\left\{\begin{array}{l}
\beta_{r}=t_{r}, t_{r} \in \mathbb{R}, r=1, \ldots, \hat{m}, \ldots, \hat{n}, \ldots, l, \\
\beta_{m}=\frac{\operatorname{sen}(n \omega)}{\operatorname{sen}((n-m) \omega)}-\frac{\sum_{\substack{r=1 \\
r \neq m, n}}^{l} t_{r} \operatorname{sen}((n-r) w)}{\operatorname{sen}((n-m) \omega)}, \\
\beta_{n}=-\frac{\operatorname{sen}(n \omega)}{\operatorname{sen}((n-m) \omega)}+\frac{\sum_{\substack{r=1 \\
r \neq m, n}}^{l} t_{r} \operatorname{sen}((m-r) w)}{\operatorname{sen}((n-m) \omega)}
\end{array}\right.
$$

e o hiperplano $\mathcal{H}_{p}^{j}$ dado por

$$
\left\{\left(\beta_{1}, \ldots, \beta_{l}\right) \in \mathbb{R}^{l}: \sum_{r=1}^{l} \beta_{r} \cos \left(r w_{j}\right)=1\right\} .
$$

Lema 3.2.1. Sejam A uma matriz constante $n \times n, p$ um inteiro, $p \geq n>2$. Se todos os autovalores de $A$ são raízes p-ésimas da unidade duas a duas distintas, então $A^{p}=I$.

Prova. Como todos autovalores da matriz $A$ são distintos, existe uma matriz não singular $N$ tal que

$$
N A N^{-1}=D
$$

onde $D$ é uma matriz diagonal cuja diagonal é constituida pelos autovalores de $A$. Daí, temos que

$$
N A^{p} N^{-1}=D^{p}
$$


Agora, como todos autovalores são raízes $p$-ésimas da unidade, temos que $D^{p}=I$. Portanto,

$$
A^{p}=I
$$

A equação característica de (3.1) é

$$
e^{\lambda l}=\sum_{r=1}^{l} \beta_{r} e^{\lambda(l-r)}
$$

ou, definindo $\mu:=e^{\lambda}$,

$$
\mu^{l}=\sum_{r=1}^{l} \beta_{r} \mu^{l-r} .
$$

Teorema 3.2.1. Se todos os zeros de (3.13) são raízes p-ésimas da unidade duas a duas distintas, então todas as soluções de (3.10) são p-periódicas.

Prova. Observamos que, fazendo

$$
\begin{aligned}
x_{1}(t) & =x(t), \\
x_{2}(t) & =x_{1}(t-1), \\
\vdots & \\
x_{l}(t) & =x_{l-1}(t-1),
\end{aligned}
$$

podemos re-escrever (3.10) na forma

$$
X(t)=M X(t-1)
$$

onde

$$
X(t)=\left(\begin{array}{c}
x_{1}(t) \\
x_{2}(t) \\
x_{3}(t) \\
\vdots \\
x_{l}(t)
\end{array}\right) \quad \text { e } \quad M=\left[\begin{array}{ccccc}
\beta_{1} & \beta_{2} & \ldots & \beta_{l-1} & \beta_{l} \\
1 & 0 & \ldots & 0 & 0 \\
0 & 1 & \ldots & 0 & 0 \\
\vdots & \vdots & \ddots & \vdots & \vdots \\
0 & 0 & \ldots & 1 & 0
\end{array}\right]
$$


$M$ é conhecida como matriz companheira do polinômio

$$
\Delta(\mu)=\mu^{l}-\sum_{r=1}^{l} \beta_{r} \mu^{l-r},
$$

veja [20] por exemplo. Note que $\operatorname{det}(M-\mu I)=\Delta(\mu)$, como $\Delta(\mu)=0$ é a equação característica (3.13), segue-se que os autovalores de $M$ são os zeros de (3.13).

Seguindo os passos de Hale em [17] para o caso bi-dimensional, dada uma função $\Phi=\operatorname{col}\left(\varphi_{1}, \ldots, \varphi_{l}\right)$, onde $\varphi_{j}$ são funções definidas de $[-1,0]$ em $\mathbb{R}$, a solução $X(t)$ de (3.14) passando por $\Phi$ é

$$
X(t)=M^{t-\theta} \Phi(\theta), \quad t=\theta, \theta+1, \ldots, \quad \text { onde } \quad \theta \in[-1,0] .
$$

De (3.15) temos que

$$
X(t+p)=M^{p} X(t)
$$

Como todos os autovalores de $M$ são raízes da unidade duas a duas distintas, pelo Lema 3.2.1 temos que $M^{p}=I$ e, portanto, $X(t+p)=X(t)$. 


\section{Exemplos}

Exemplo 3.2.1. Tomemos $l=2$ em (3.10), isto é,

$$
x(t)=\beta_{1} x(t-1)+\beta_{2} x(t-2) .
$$

Como a curva de bifurcação $\Gamma$ de (3.16), correspondente à hipersuperfície (3.11) é dada por

$$
\left\{\begin{array}{l}
\beta_{1}=2 \cos w, \\
\beta_{2}=-1, \quad w \in(0, \pi)
\end{array}\right.
$$

e, dado $p>2$ inteiro, a reta $\mathcal{H}_{p}^{j}, j \in \mathcal{J}_{p}$, correspondente ao hiperplano (3.11) é dada por

$$
\left\{\left(\beta_{1}, \beta_{2}\right) \in \mathbb{R}^{2}: \beta_{1} \cos w_{j}+\beta_{2} \cos \left(2 w_{j}\right)=1\right\}
$$

temos que

$$
\Gamma \cap \mathcal{H}_{p}^{j}=\left\{\left(\beta_{1}, \beta_{2}\right) \in \mathbb{R}^{2}:\left(\beta_{1}, \beta_{2}\right)=\left(2 \cos w_{j},-1\right)\right\}, \quad j \in \mathcal{J}_{p}
$$

Assim, observamos que:

a) Pelo Teorema 3.1.1, a Equação (3.16), $\operatorname{com} \beta_{1}=2 \cos w_{j}$ e $\beta_{2}=-1$, tem soluções $p$-periódicas, $p>2$ inteiro e a matriz citada na prova do Teorema 3.2.1 é dada por

$$
M=\left[\begin{array}{cc}
2 \cos w_{j} & -1 \\
1 & 0
\end{array}\right] .
$$

As raízes características são $\lambda_{1}=\cos w_{j}+i \operatorname{sen} w_{j}$ e $\lambda_{2}=\cos w_{j}-i \operatorname{sen} w_{j}$, e para cada $j \in \mathcal{J}_{p}$ elas são duas raízes $p$-ésimas da unidade. O Lema 3.2.1 implica $M^{p}=I$. Logo, pelo Teorema 3.2.I todas soluções são $p$-periódicas. 
b) O Teorema 3.1 .1 garante que se $\beta_{1}+\beta_{2}=1$, então qualquer função 1periódica é solução de (3.16). Mas, neste caso, nem todas as soluções de (3.16) são 1-periódicas. De fato, basta verificarmos que

$$
x(t)=\left(-\beta_{2}\right)^{t}
$$

é solução não periódica, se $\beta_{2} \neq 0$ ou $\beta_{2} \neq-1$.

c) O Teorema 3.1.1 nos diz que se $\beta_{2}=\beta_{1}+1,(3.16)$ tem solução 2-periódica da forma $x(t)=a(t) \cos \pi t$, qualquer que seja $a(t)$ 1-periódica. Mas, nem toda solução dessa equação é dessa forma, basta verificarmos que

$$
x(t)=\beta_{2}^{t}
$$

é solução não periódica, se $\beta_{2} \neq 0$ ou $\beta_{2} \neq 1$.

Exemplo 3.2.2. Para $l=3$, temos

$$
x(t)=\beta_{1} x(t-1)+\beta_{2} x(t-2)+\beta_{3} x(t-3) .
$$

A superfície de bifurcação de (3.17), correspondente à hipersuperfície (3.11) pode ser parametrizada por

$$
\Gamma:\left\{\begin{array}{l}
\beta_{1}=2 \cos w+t_{3}, \\
\beta_{2}=-1-(2 \cos w) t_{3} \\
\beta_{3}=t_{3}, \quad w \in(0, \pi) \text { e } t_{3} \in \mathbb{R}
\end{array}\right.
$$

e, dado $p>2$ inteiro, o plano $\mathcal{H}_{p}^{j}, j \in \mathcal{J}_{p}$, correspondente ao hiperplano (3.11) é dado por

$$
\left\{\left(\beta_{1}, \beta_{2}, \beta_{3}\right) \in \mathbb{R}^{3}: \beta_{1} \cos w_{j}+\beta_{2} \cos \left(2 w_{j}\right)+\beta_{3} \cos \left(3 w_{j}\right)=1\right\} .
$$

Se $t_{3} \in \mathbb{R}$ e $j \in \mathcal{J}_{p}$, temos que

$$
\Gamma \cap \mathcal{H}_{p}^{j}=\left\{\left(\beta_{1}, \beta_{2}, \beta_{3}\right) \in \mathbb{R}^{3}:\left(\beta_{1}, \beta_{2}, \beta_{3}\right)=\left(2 \cos w_{j}+t_{3},-1-2 t_{3} \cos w_{j}, t_{3}\right)\right\}
$$


Assim, se $\left(\beta_{1}, \beta_{2}, \beta_{3}\right) \in \Gamma \cap \mathcal{H}_{p}^{j}$, a matriz citada na prova do Teorema 3.2 .1 é

$$
M=\left[\begin{array}{ccc}
2 \cos w_{j}+t_{3} & -1-2 t_{3} \cos w_{j} & t_{3} \\
1 & 0 & 0 \\
0 & 1 & 0
\end{array}\right]
$$

e seu polinômio característico é

$$
\Delta(\mu)=-\left(\mu-t_{3}\right)\left(\mu^{2}-2 \mu \cos w_{j}+1\right)
$$

cujas raízes são

$$
\mu_{1}=t_{3}, \quad \mu_{2}=\bar{\mu}_{2}=\cos w_{j}+i \operatorname{sen} w_{j} .
$$

Observe que se $t_{3}= \pm 1$, os $\mu_{j}$ 's são raízes $p$-ésimas da unidade. Assim, pelo Lema 3.2.1 temos que $M^{p}=I$. Portanto, se $\left(\beta_{1}, \beta_{2}, \pm 1\right) \in \Gamma \cap \mathcal{H}_{p}^{j}$, o Teorema 3.2.1 garante que todas as soluções de (3.17) são $p$-periódicas.

A figura a seguir mostra a superfície de bifurcação para a equação (3.17).

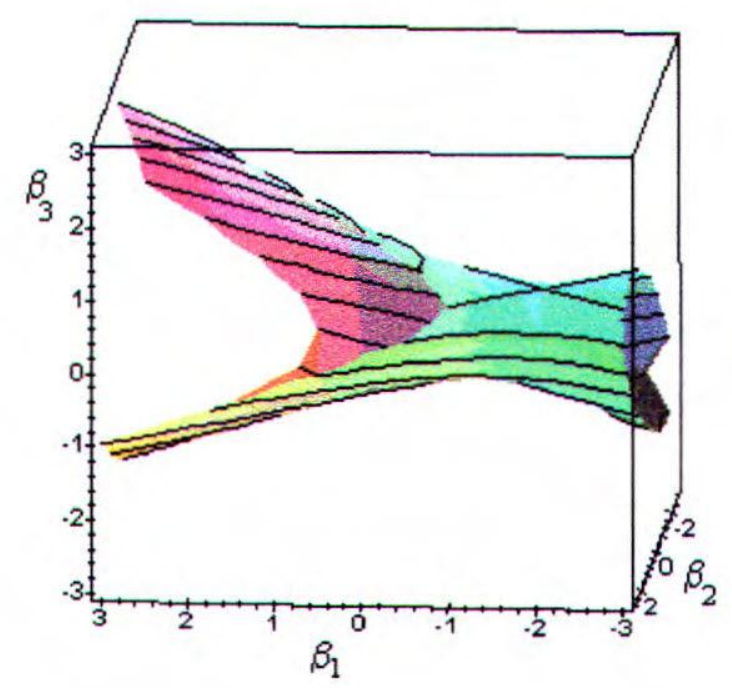

fig 3.2 . 
Exemplo 3.2.3. Brand [3], fez uma análise do comportamento das soluções da equação

$$
x_{n+1}=\frac{a x_{n}+b}{c x_{n}+d}, \quad n=0,1, \ldots
$$

onde $a, b, c$ são constantes reais tais que $a d-b c \neq 0$ e $c \neq 0$.

Os resultados de Brand também podem ser encontrados em [22].

Seja a equação

$$
x(t)=\frac{a x(t-1)+b}{c x(t-1)+d}, \quad c \neq 0 \text { ead }-b c \neq 0 .
$$

Introduzindo como em [3] a mudança de variáveis $x(t)=y(t)-d / c$, podemos escrever (3.18) na forma

$$
y(t)=\beta_{1}+\frac{\beta_{2}}{y(t-1)}, \quad y(t) \neq 0,
$$

onde $\beta_{1}=\frac{a+d}{c}$ e $\beta_{2}=-\frac{a d-b c}{c^{2}}$.

Ainda por inspiração em [3], com uma nova mudança de variáveis, $y(t)=$ $z(t) / z(t-1)$, re-escrevemos (3.19) na forma

$$
z(t)=\beta_{1} z(t-1)+\beta_{2} z(t-2)
$$

Observe que $x(t)$ é $p$-periódica, se $z(t)$ é $p$-periódiça.

Os Teoremas 3.1.1 e 3.2.1 proporcionam um enfoque distinto para o contexto de Brand. A análise em [3] depende das raízes características da Equação (3.20), enquanto a nossa está baseada nos coeficientes. Além disso, conseguimos exibir aqui as expressões das soluções periódicas obtidas. Obtemos as seguintes consequências:

a) Toda função 1-periódica é solução de (3.18) se,

$$
(a+d) c-(a d-b c)=1
$$


b) A Eq.(3.18) tem solução 2-periódica da forma

$$
x(t)=a_{1}(t) \cos (\pi t), \quad a_{1}(t) \quad \text { 1-periódica, }
$$

se,

$$
(a+d) c+a d-b c=-1
$$

c) A Equação (3.18) tem soluções 2-periódicas da forma

$$
x(t)=a_{0}(t)+a_{1}(t) \cos (\pi t), \quad a_{0}(t), a_{1}(t) \quad \text { 1-periódicas },
$$

se

$$
a+d=0 .
$$

d) Suponha que $a+b \neq 0$. Todas as soluções de (3.18) são da forma

$$
x_{p}^{j}(t)=a_{j}(t) \cos \left(w_{j} t\right)+b_{j}(t) \operatorname{sen}\left(w_{j} t\right), \quad j \in \mathcal{J}_{p},
$$

onde $a_{j}(t), b_{j}(t)$ são funções 1-periódicas, se

$$
a c-b c=c^{2} \quad \text { e } \quad a+d=2 c \cos w_{j}, \quad c \neq 0, \quad j \in \mathcal{J}_{p} .
$$

Portanto, neste caso, todas as soluções de (3.18) são p-periódicas. 


\section{Capítulo 4}

\section{Um Estudo da Região de Estabilidade}

Os resultados dos Capítulos 2 e 3, os trabalhos de Carvalho [4], Clark [9], Levin e May [24] e Kuruklis [23], citados na Seção 1.3.2 sugerem uma interessante área de investigação, a saber, o estudo da perturbação singular da equação

$$
x(t)=\sum_{r=1}^{l} \alpha_{r} x(t-r),
$$

com relação à região de estabilidade no espaço dos parâmetros, isto é, estudar o problema

$$
\varepsilon \dot{x}(t)+x(t)=\sum_{r=1}^{l} \alpha_{r} x(t-r),
$$

quando $\varepsilon \rightarrow 0$, no espaço dos parâmetros $\alpha_{r}$ 's. Observando o Exemplo 3.2.2 notamos que, no caso geral, este problema não é trivial. Motivados pelo Exemplo 2.3.2 nos propomos a estudar o seguinte caso particular de (2.6),

$$
\varepsilon \dot{x}(t)+x(t)=\alpha_{1} x(t-1)+\alpha_{l} x(t-l),
$$

isto é, fazemos $\alpha_{0}=-1, \alpha_{r}=0, r=2, \ldots, l-1, l \geq 2$ em (2.6). 
Observamos que (1.28) é uma versão discretizada de (4.1) para o caso $\varepsilon=0$, $t+1=n \in \mathbb{N}, \alpha_{1}=a, \alpha_{l}=-b$ e $l=k+1$. Note que a equação característica associada à (4.1) é dada por

$$
\Delta\left(\varepsilon, \alpha_{1}, \alpha_{l} ; \lambda\right)=\varepsilon \lambda+1-\alpha_{1} e^{-\lambda}-\alpha_{l} e^{-l \lambda}=0 .
$$

\subsection{Estabilidade}

Os resultados a seguir nos fornecem informações importantes acerca da região de estabilidade de (4.1).

Proposiçāo 4.1.1. Se $\left|\alpha_{1}\right|+\left|\alpha_{l}\right|<1$, então todas as raízes de (4.2) têm parte real negativa.

Prova. De (4.2) temos que

$$
\Re(\Delta(\lambda))=1+\varepsilon \Re(\lambda)-\alpha_{1} e^{-\Re(\lambda)} \cos \Im(\lambda)-\alpha_{l} e^{-l \Re(\lambda)} \cos (l \Im(\lambda))=0 .
$$

Vamos supor, por contradição, que (4.2) tenha uma rajz tal que $\Re(\lambda) \geq 0$. Temos que

$$
1 \leq|1+\varepsilon \Re(\lambda)|=\left|\alpha_{1} e^{-\Re(\lambda)} \cos \Im(\lambda)+\alpha_{l} e^{-l \Re(\lambda)} \cos (l \Im(\lambda))\right| \leq\left|\alpha_{1}\right|+\left|\alpha_{l}\right|<1
$$

e esta contradição prova nossa proposição.

As curvas $\Gamma_{s k}^{\varepsilon}(l), k \in \mathbb{Z}$, correspondentes às hipersuperfícies (2.25) para (4.1) são

$$
\Gamma_{s k}^{\varepsilon}(l):\left\{\begin{array}{l}
\alpha_{1}=\frac{-\varepsilon(2 k \pi-w) \cos (l w)+\operatorname{sen}(l w)}{\operatorname{sen}((l-1) w)} \\
\alpha_{l}=\frac{\varepsilon(2 k \pi-w) \cos w-\operatorname{sen} w}{\operatorname{sen}((l-1) w)},
\end{array}\right.
$$

onde $\omega \in\left(\frac{(l-1) k+s-1}{l-1} \pi, \frac{(l-1) k+s}{l-1} \pi\right), s=1, \ldots, l-1$.

Cada uma dessas curvas particiona o plano $\alpha_{1} \alpha_{l}$ em duas ou mais regiões conexas. 
Se $A_{s k}^{\varepsilon}(l)$ é a região conexa do plano $\alpha_{1} \alpha_{l}$ que contém a origem, definimos

$$
R_{s k}^{\varepsilon}(l)=\left\{\begin{array}{l}
A_{s k}^{e}(l) \cap\left\{\left(\alpha_{1}, \alpha_{l}\right): \alpha_{1}+\alpha_{l}<1\right\}, \text { se } \varepsilon>0, \\
A_{s k}^{\varepsilon}(l) \cap\left\{\left(\alpha_{1}, \alpha_{l}\right): \alpha_{1}+\alpha_{l}<1 \text { e } \alpha_{1}>(-1)^{l} \alpha_{l}-1\right\}, \text { se } \varepsilon=0 .
\end{array}\right.
$$

Proposiçāo 4.1.2. Se $\varepsilon \geq 0$ el $l \geq 2$ inteiro, então

$$
\mathcal{R}_{l}^{\varepsilon}=\bigcap_{k \in \mathbf{Z}} \bigcap_{s=1}^{l-1} R_{s k}^{\varepsilon}(l)
$$

é a região de estabilidade de (4.1).

Prova. Pela Proposição 4.1.1 temos que, se $\left(\alpha_{1}, \alpha_{l}\right)$ é tal que $\left|\alpha_{1}\right|+\left|\alpha_{l}\right|<$ 1, todas as raízes de (4.2) têm parte real negativa. Logo, pelo Teorema 1.3.1 (continuidade das raízes com relação aos parâmetros) se $\left(\alpha_{1}, \alpha_{l}\right)$ variar no plano $\alpha_{1} \alpha_{l}$ a partir dessa vizinhança, aparecerá uma raiz com parte real positiva se, somente se, $\left(\alpha_{1}, \alpha_{l}\right)$ cruzar uma curva de bifurcação ou a reta $\Delta\left(0, \varepsilon, \alpha_{1}, \alpha_{l}\right)=$ $\alpha_{1}+\alpha_{l}-1=0$.

Observamos que para o caso $\varepsilon=0$ as curvas $\Gamma_{s k}^{0}$ não dependem de $k$. Assim, representaremos $\Gamma_{s k}^{0}, A_{s k}^{0}$ e $R_{s k}^{0}(l)$ por $\Gamma_{s}^{0}, A_{s}^{0}$ e $R_{s}^{0}(l)$, respectivamente.

Vamos agora estudar a forma assintótica da região de estabilidade de (4.1), quando $\varepsilon \rightarrow 0$. Nesta análise nos restringiremos ao caso:

$$
\varepsilon \dot{x}(t)+x(t)=\alpha_{1} x(t-1)+\alpha_{2} x(t-2) .
$$

A região $\mathcal{R}_{2}^{0}$ é o interior do triângulo determinado pelo segmento $\Gamma$ do plano $\alpha_{1} \alpha_{2}$ dado pela parametrização

$$
\Gamma(w):=\Gamma_{1}^{0}:\left\{\begin{array}{l}
\alpha_{1}(w)=2 \cos w \\
\alpha_{2}(w)=-1, \quad \omega \in[0, \pi]
\end{array}\right.
$$

e as retas $r_{1}, r_{2}$ ligando os extremos de $\Gamma$ ao ponto $(0,1)$ (veja a Figura 4.1 para $\varepsilon=0)$. 
Este fato é bem conhecido, ver [22] ou [23].

Para simplificar a notação representaremos $\Gamma_{1, k}^{\varepsilon}(2)(w)$ por $\Gamma_{k}^{\varepsilon}(w)$.

O teorema a seguir descreve as parametrizações $\Gamma_{k}^{\varepsilon}(w), w \in(0, \pi)$, das curvas $\Gamma_{k}^{\varepsilon}$, estabelecendo que, dado $\delta>0$, para um $\varepsilon>0$ suficientemente pequeno, se $w \in[\delta, \pi-\delta], k>0$, o ponto $\Gamma_{k}^{\varepsilon}(w)$ acompanha proximamente o ponto $\Gamma(w)$ do segmento $\Gamma$, isto é, $\left|\Gamma_{k}^{e}(w)-\Gamma(w)\right|$ pode ser feito arbitrariamente pequeno. Além disso, $\Gamma_{k}^{\varepsilon}(w) \rightarrow(-\infty, \infty)$, com $w \rightarrow 0$ e $\Gamma_{k}^{\varepsilon}(w) \rightarrow(-\infty,-\infty)$, com $w \rightarrow \pi$ e vetor $\tau(w):=\frac{d}{d w}\left(\Gamma_{k}^{\varepsilon}(w)\right)$, tangente à $\Gamma_{k}^{\varepsilon}(w)$, tende à direção da reta $r_{1}$ ou $r_{2}$ quando $w \rightarrow 0$ ou $w \rightarrow \pi$, respectivamente.

No caso $k<0$, o comportamento de $\Gamma_{k}^{e}(w)$ é análogo. Basta substituir $\Gamma(w)$ por $-\Gamma(w)$.

Isto significa que as curvas $\Gamma_{k}^{\varepsilon}(w), w \in(0, \pi), \equiv>0$, têm as formas esboçadas abaixo

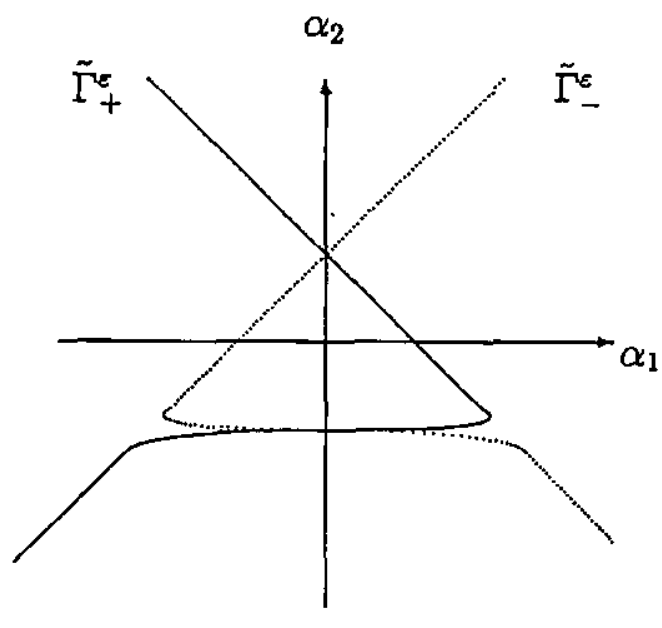

$$
\varepsilon>0, \text { pequeno }
$$

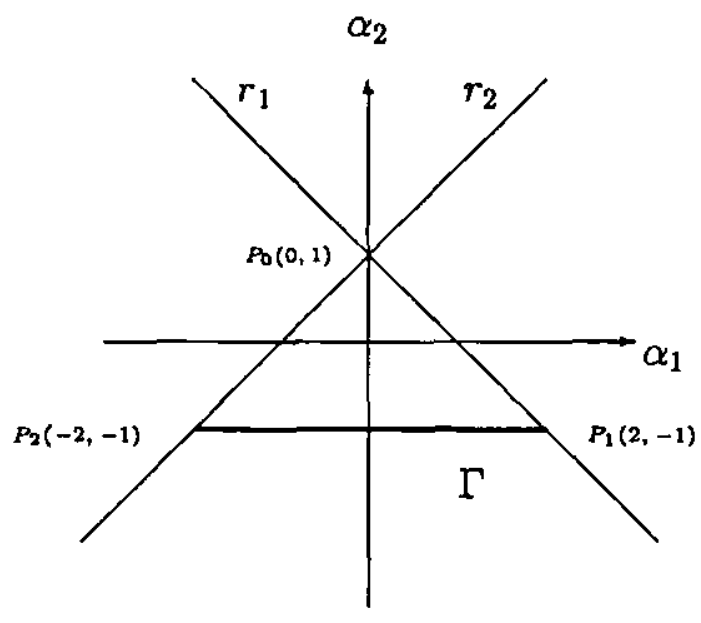

$\varepsilon=0$

Figura 4.1: As curvas $\Gamma_{k}^{\varepsilon}, \varepsilon>0$ pequeno, estão representadas por $\tilde{\Gamma}_{+}^{\varepsilon}$, se $k>0$ e $\tilde{\Gamma}_{-}^{\varepsilon}$, se $k \leq 0$. 
Teorema 4.1.1. Sejam $J \subset(0, \pi)$ um intervalo fechado.

$$
\Gamma_{k}^{\varepsilon}(w) \rightarrow \Gamma(w)
$$

uniformemente em $J$, quando $\varepsilon \rightarrow 0$. Além disso, o vetor tangente à curva $\Gamma_{k}^{\varepsilon}$ no ponto $\Gamma_{k}^{\varepsilon}(w)$ tende à direção da reta $r_{1}$ ou $r_{2}$ quando $w \rightarrow 0$ ou $w \rightarrow \pi$, respectivamente.

Prova. Como já vimos no Exemplo 2.3.2 as k-ésimas hipersuperfícies (curvas) de bifurcação são dadas por

$$
\Gamma_{k}^{\varepsilon}(w):\left\{\begin{array}{l}
\alpha_{1}(w)=-\frac{\varepsilon(2 k \pi-\omega) \cos (2 \omega)}{\operatorname{sen} \omega}+2 \cos \omega, \\
\alpha_{2}(w)=\frac{\varepsilon(2 k \pi-\omega) \cos w}{\operatorname{sen} \omega}-1, \quad \omega \in(0, \pi) .
\end{array}\right.
$$

Seja $\hat{\Gamma}(\delta)$ a vizinhança de $\Gamma$ dada por

$$
\hat{\Gamma}(\delta)=\left\{\left(\alpha_{1}, \alpha_{2}\right): \operatorname{dist}\left(\left(\alpha_{1}, \alpha_{2}\right), \Gamma\right)<\delta\right\} .
$$

Primeiro vamos mostrar $\Gamma_{k}^{\varepsilon}(w) \in \hat{\Gamma}(\delta)$ se $w \in[\delta, \pi-\delta]$, isto é, vamos mostrar que dado $\delta>0$ pequeno, existe $\hat{\varepsilon}>0$ tal que para todo $0<\varepsilon<\hat{\varepsilon}$,

$$
\left\|\left(\alpha_{1}(w)-2 \cos w, \alpha_{2}(w)+1\right)\right\|<\delta, \quad w \in[\delta, \pi-\delta] .
$$

Para isso, observe que dado $\delta>0$, existe $\hat{\varepsilon}_{1}>0$ tal que se $0<\varepsilon<\hat{\varepsilon}_{1}$, então

$$
\left|\varepsilon(2 k \pi-w) \frac{\cos (2 w)}{\operatorname{sen} w}\right|<\frac{\delta}{\sqrt{2}}, \quad w \in[\delta, \pi-\delta] .
$$

De fato, existe $M>0$ tal que $\left|(2 k \pi-w) \frac{\cos (2 w)}{\operatorname{sen} w}\right| \leq M$, para todo $w \in[\delta, \pi-\delta]$. Se tomarmos $\varepsilon<\frac{\delta}{\sqrt{2} M}$ temos que

$$
\begin{aligned}
\left|\varepsilon(2 k \pi-w) \frac{\cos (2 w)}{\operatorname{sen} w}\right| & =\varepsilon\left|(2 k \pi-w) \frac{\cos (2 w)}{\operatorname{sen} w}\right| \\
& <\frac{\delta}{\sqrt{2} M}\left|(2 k \pi-w) \frac{\cos (2 w)}{\operatorname{sen} w}\right|<\frac{\delta}{\sqrt{2}} .
\end{aligned}
$$


Analogamente, para este mesmo $\delta$, existe $\hat{\varepsilon}_{2}>0$ tal que se $0<\varepsilon<\hat{\varepsilon}_{2}$, então

$$
\left|\varepsilon(2 k \pi-w) \frac{\cos w}{\operatorname{sen} w}\right|<\frac{\delta}{\sqrt{2}}, \quad w \in[\delta, \pi-\delta] .
$$

Assim, para todo $\varepsilon>0$ tal que $\varepsilon<\hat{\varepsilon}=\min \left\{\hat{\varepsilon}_{1}, \hat{\varepsilon}_{2}\right\}$ temos

$$
\begin{aligned}
\left\|\left(\alpha_{1}(w)-2 \cos w, \alpha_{2}(w)+1\right)\right\|^{2}= \\
=\left\|\left(-\frac{\hat{\varepsilon}(2 k \pi-\omega) \cos (2 \omega)}{\operatorname{sen} \omega}, \frac{\hat{\varepsilon}(2 k \pi-\omega) \cos w}{\operatorname{sen} \omega}\right)\right\|^{2} \\
<\left(\frac{\delta}{\sqrt{2}}\right)^{2}+\left(\frac{\delta}{\sqrt{2}}\right)^{2}=\delta^{2} . \quad w \in[\delta, \pi-\delta] .
\end{aligned}
$$
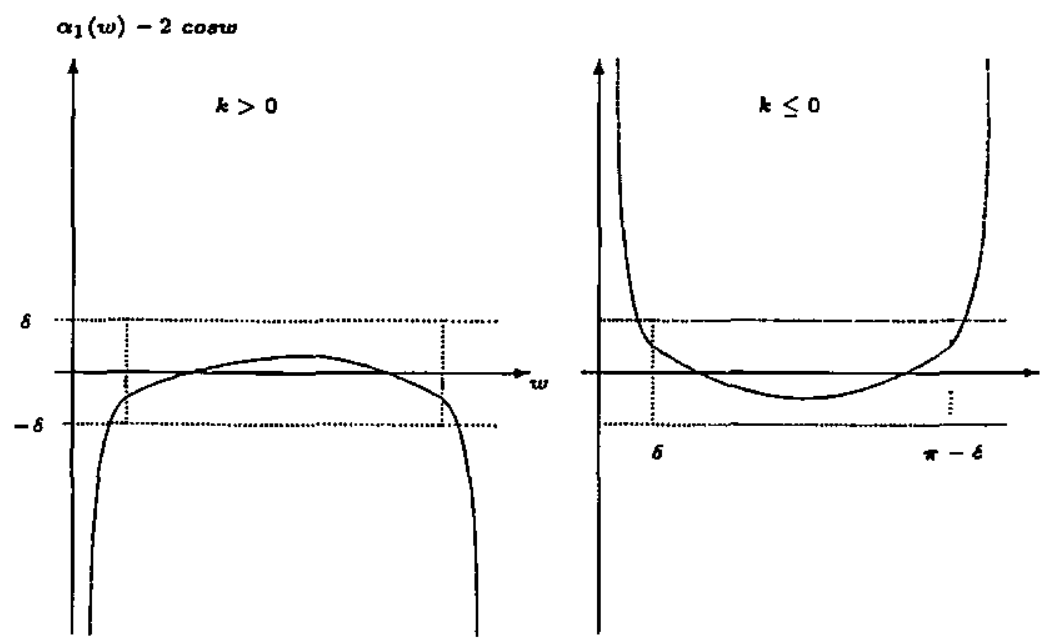

Figura 4.2: A curva $\alpha_{1}(w)-2 \cos w$ para $\varepsilon$ pequeno.

Logo, dado um intervalo fechado $J \subset(0, \pi)$ qualquer, para $\delta>0$ suficientemente pequeno, podemos tomar $0<\varepsilon<\hat{\varepsilon}$, tal que $J \subset[\delta, \pi-\delta]$.

Concluindo, mostramos que para qualquer interralo fechado $J \subset(0, \pi)$ podemos encontrar um intervalo $(\delta, \pi-\delta)$ que contém o $J$ e a curva $\Gamma_{k}^{\varepsilon}(w)$ está na vizinhança $\hat{\Gamma}(\delta)$ para $w \in[\delta, \pi-\delta]$. 


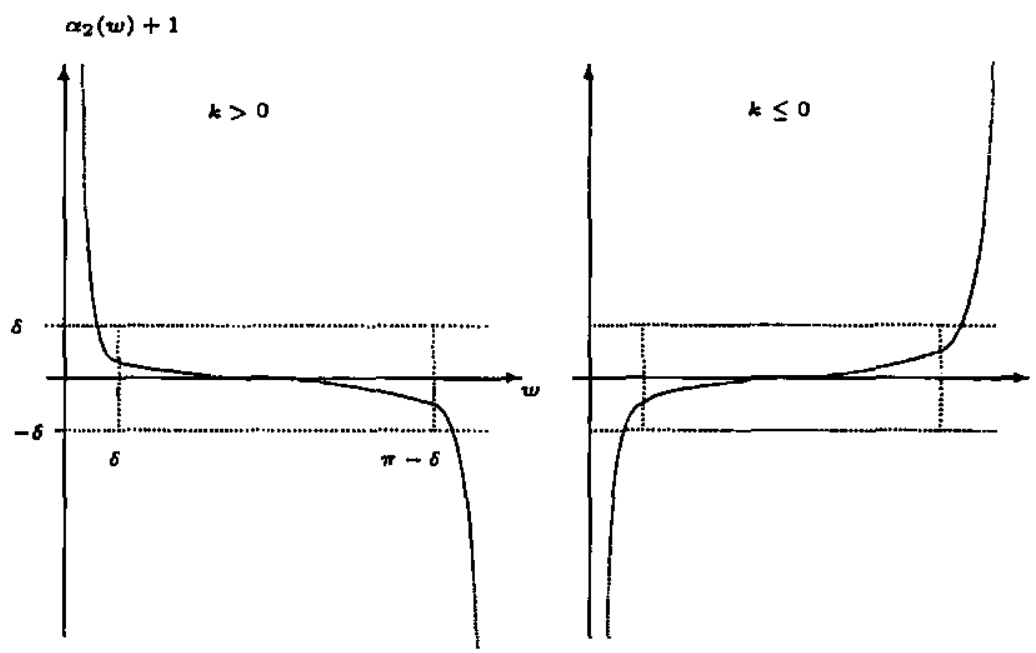

Figura 4.3: A curva $\alpha_{2}(w)+1$ para $\Xi$ pequeno.

Agora, vamos considerar $\tau(w)$ um vetor tangente à curva $\Gamma_{k}^{\varepsilon}$, no ponto $\Gamma_{k}^{\varepsilon}(w)$.

$$
\begin{aligned}
\tau(w)= & \left(\varepsilon \frac{\cos (2 w)}{\operatorname{sen} w}+\varepsilon(2 k \pi-w) \frac{2 \operatorname{sen}(2 w) \operatorname{sen} w+\cos (2 w) \cos w}{\operatorname{sen}^{2} w}-2 \operatorname{sen} w\right. \\
& \left.-\varepsilon \frac{\cos w}{\operatorname{sen} w}-\varepsilon(2 k \pi-w) \frac{1}{\operatorname{sen}^{2} w}\right)
\end{aligned}
$$

ou

$$
\begin{aligned}
\tilde{\tau}(w) & =\left(\operatorname{sen}^{2} w\right) \tau(w)= \\
& \left(\varepsilon \cos (2 w) \operatorname{sen} w+\varepsilon(2 k \pi-w)(2 \operatorname{sen}(2 w) \operatorname{sen} w+\cos (2 w) \cos w)-2 \operatorname{sen}^{3} w\right. \\
& -\varepsilon \cos w \operatorname{sen} w-\varepsilon(2 k \pi-w)) .
\end{aligned}
$$

Observe que $\tilde{\tau}$ é um múltiplo escalar do vetor $\tau(w)$ para cada $w \in(0, \pi)$. Se $k>0$ e $w \rightarrow 0$ temos que

$$
\tilde{\tau}(w)=(2 k \pi \varepsilon,-2 k \pi \varepsilon)=2 k \pi \varepsilon(1 .-1)
$$

$\mathrm{E}$, se $k<0$ temos

$$
\tilde{\tau}(w)=(-2(k-1 / 2) \pi \varepsilon,-2(k-1 / 2) \pi \varepsilon)=2\left(k_{1}+1 / 2\right) \varepsilon \pi(1,1) \quad k_{1}=-k>0
$$

quando $w \rightarrow \pi$. 
4.1 Estabilidade

69

Os resultados obtidos neste capítulo sugerem o seguinte:

Observaçāo 4.1.1. Embora os resultados obtidos neste capítulo indiquem que a região de estabilidade, $\mathcal{R}_{2}^{\varepsilon}$, de (4.1) aproxima a região de estabilidade $\mathcal{R}_{2}^{0}$, da equação limite, não se pode estabelecer nenhuma relação de inclusão entre $\mathcal{R}_{2}^{\epsilon} e$ $\mathcal{R}_{2}^{0}$, para $\varepsilon \rightarrow 0$.

De fato, uma análise das curvas $\Gamma_{k}^{\varepsilon}, \varepsilon>0$ pequeno, $k \in \mathbb{Z}$, mostra que os vértices $P_{1}$ e $P_{2}$ (veja Fig.4.1) estão fora de $\mathcal{R}_{2}^{\varepsilon}$ como se deduz da Figura 4.3. Por outro lado, o ponto $(0,-1) \in \partial \mathcal{R}_{2}^{\varepsilon}$ é interior à $\mathcal{R}_{2}^{\Sigma}, \varepsilon>0$. 


\section{Referências Bibliográficas}

[1] Bellman, R. \& K. Cooke. Differential-Difference Equations. Academic Press, New York, 1963.

[2] Beuter, A., Bélair. \& Labrie, C. Feedback and delays in neurological diseases: A modeling study using dynamical systems. Bull. Math. Biol. 55, 525-541, 1993.

[3] Brand, L., A sequence defined by a difference equation. Amer. Math. Monthly, 62, 489-492, 1955.

[4] Carvalho, L.A.V. On Liapunov Functionals for Linear Difference Equations. Ph.D. Thesis, Brown University, Providence, R.I., 1979.

[5] Carvalho, L.A.V. On a method to investigate bifurcation of periodic solutions in retarded differential equations. Preprint, ICMSC-USP, São Carlos, 1993.

[6] Carvalho, L. A. V. and Ladeira, L. A. C. On Periodic Orbits of Autonomous Differential-Difference Equations. Differential Equations and Applications to Biology and to Industry-Proceedings of the June 1-4, 1994, Claremont International Conference dedicated to memory of Stavros Busenberg, Edited by M. Martelli et al., World Scientific, pp. 57-64, 1996. 
[7] Carvalho, L.A.V., K.L. Cooke and L. A. C. Ladeira. On Periodic Solutions for a Class of Linear Scaled Differential Equations - Part I. Preprint in Pomona College, Claremont, CA, 1996.

[8] Carvalho, L.A.V., K.L. Cooke and L. A. C. Ladeira. On Periodic Solutions for a Class of Linear Scaled Differential Equations - Part II. Preprint in Pomona College, Claremont, CA, 1996.

[9] Clark, C. W. A delayed recruitment model of population dynamics with an application to baleen whale populations. J. Math. Biol. 3, 381-391, 1976.

[10] Cruz, J. H. and P. Z. Táboas. Periodics solutions of a Linear Scalar Differential-Difference Equation. Preprint, ICMSC-LSP, São Carlos, 1995.

[11] Cruz, J. H. e P. Z. Táboas. Soluções Periódicas Para Um Problema de Perturbação Singular Com Finitos Retardos. Atas do 45ः SBA, 667-673, Florianópolis, SC, 1997.

[12] Diekmann, O., S. A. van Gils, S. M. V. Lunel; W. Hans-Otto. Delay equations. Functional, complex, and nonlinear analysis. Applied Mathematical Sciences, 110. Springer-Verlag, New York, 1995.

[13] Dieudonné, J. Fundamentos de Analisis Moderno. Ed. Reverté, S. A., 1966.

[14] Driver, R. D., D. W. Sasser and M. L. Slater. The equation $x^{\prime}(t)=$ $a x(t)+b x(t-\tau)$ with "small" delay, Amer. Math. Monthly, 80, 1973, 990-995. 
[15] El'sgol'ts, L. E. Introduction to the Theory of Differential Equations with Deviating Arguments. Holden-Day, San Francisco, 1966.

[16] Ferreira, R. R. Métodos Diretos Para a Análise do Comportamento Assintótico de Equações Diferenciais Funcionais e Equações a Diferenças Finitas. Tese de Doutorado, ICMSC-USP, São Carlos, 1986.

[17] Hale, J. K. and S. M. V. Lunel Introduction to Functional Differential Equations. Applied Mathematical Sciences, Vol. 99, Springer-Verlag, New York, 1993.

[18] Hale, J. K. and W. Huang. Global geometry of stable regions for two delay differential equations. J. Math. Anal. Appl. 178, 3-11-362, 1993.

[19] Hayes, N. Roots of the transcendental equation associated with a certain differential difference equation. J. London Math. Soc. 25, 226-232, 1950.

[20] Horn, Roger A. Matrix Analysis. Cambridge Liniversity Press, Cambridge, 1991.

[21] Hurt, J., Some Aspects of Difference Equations. Ph.D. Thesis, Brown University, Providence, RI, 1967.

[22] Kocic, V. L. and G. Ladas. Global Behavior of Nonlinear Difference Equations of Higher Order with Applications. Math. and Its Applications, Vol.256, Kluwer Acad. Publishers, Netherlands, 1993.

[23] Kuruklis, S. A. The Asymptotic Stability of $x_{n+1}-a x_{n}+b x_{n-k}=0$. Journal of Math. Analysis and Applications, 188, 719-731, 1994.

[24] Levin, S. A. and May, R. A note on difference delay equations. Theor. Pop. Biol. 9, 178-187, 1976. 
[25] Mahaffy, J. M. and K. M. Joiner. A Geometric Analysis of Stabǐlity Regions for a linear Differential Equations with two Delays.International Journal of Bifurcation and Chaos, Vol. 5, No. 3, 779-796, 1995.

[26] Melvin, W. R.. Stability Propries of Functional Differential Equations. J. Math. Anal. Appl. Vol. 48, 1974.

[27] Mizumo, M. and K. Ikeda, An unstable mode selection rule: frustrated optical instability due to two competing boundary conditions. Physica D, 36, 327-342, 1989.

[28] Nussbaum, R. Differential delay equations with two time lags. Mem. Amer. Math. Soc., 16, 1-62, 1978.

[29] Philos, CH, G. Asymptotic Behavior, Nonoscillation and Stability in Periodic First Order Linear Delay Differential Equations. Preprint, Dept. of Math., Univ. of Ioannima, Greece, 1997.

[30] Sharkovsky, A. N., L. Y. Maistrenko and E. Y. Romanenko. Difference Equations and Applications. Math. and Its Applications, Vol. 250, Kluwer Acad. Publishers, Netherlands, 1993.

[31] Tanaka, S. M. Um estudo sobre soluções periódicas de equaçōes diferenciais funcionais. Tese de Doutorado, ICMSC-L'SP-São Carlos, 1994. 